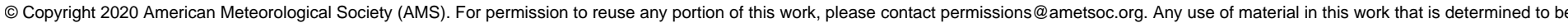

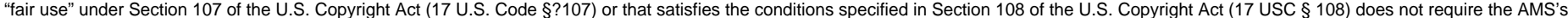

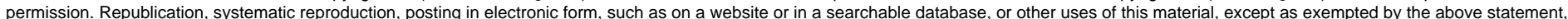

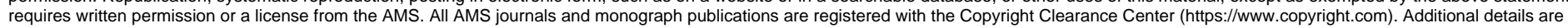
provided in the AMS Copyright Policy statement, available on the AMS website (https://www.ametsoc.org/PUBSCopyrightPolicy).

\title{
Normal Mode Spectra of Idealized Baroclinic Waves
}

\author{
Matthew R. Ambacher And Michael L. Waite \\ University of Waterloo, Waterloo, Ontario, Canada
}

(Manuscript received 24 May 2019, in final form 13 December 2019)

\begin{abstract}
Normal modes are used to investigate the contributions of geostrophic vortices and inertia-gravity waves to the energy spectrum of an idealized baroclinic wave simulation. The geostrophic and ageostrophic modal spectra (GE and AE, respectively) are compared to the rotational and divergent kinetic energy (RKE and DKE, respectively), which are often employed as proxies for vortex and wave energy. In our idealized $f$-plane framework, the horizontal modes are Fourier, and the vertical modes are found by solving an appropriate eigenvalue problem. For low vertical mode number $n$, both the GE and AE spectra are steep; however, for higher $n$, while both spectra are shallow, the AE is shallower than the GE and the spectra cross. The AE spectra are peaked at the Rossby deformation wavenumber $k_{n}^{R}$, which increases with $n$. Analysis of the horizontal mode equations suggests that, for large wavenumbers $k \gg k_{n}^{R}$, the GE is approximated by the RKE, while the AE is approximated by the sum of the DKE and potential energy. These approximations are supported by the simulations. The vertically averaged RKE and DKE spectra are compared to the sum of the GE and AE spectra over all vertical modes; the spectral slopes of the GE and AE are close to those of the RKE and DKE, supporting the use of the Helmholtz decomposition to estimate vortices and waves in the midlatitudes. However, the AE is consistently larger than the DKE because of the contribution from the potential energy. Care must be taken when diagnosing the mesoscale transition from the intersection of the vortex and wave spectra; GE and AE will intersect at a different scale than RKE and DKE, despite their similar slopes.
\end{abstract}

\section{Introduction}

In the decades since Nastrom and Gage (1985) first described the shape of the atmospheric kinetic energy (KE) spectrum, the mystery of this spectrum has motivated fundamental research in several areas of geophysical fluid dynamics. The -3 spectral slope at synoptic scales is well explained by quasigeostrophic (QG) turbulence theory (Charney 1971), but the dynamical mechanisms behind the mesoscale $-5 / 3$ slope, which extends from scales of $O(100)$ to $O(1) \mathrm{km}$, is still an active area of research. While some early work focused on the possibility of an inverse energy cascade through the mesoscale driven by convection (Gage 1979; Lilly 1983), it now appears that the energy cascade is downscale at sub-100-km scales (Lindborg and Cho 2001). Different mechanisms have been proposed for such a cascade, including inertia-gravity waves (IGWs; Dewan 1979; VanZandt 1982; Bartello 1995), anisotropic stratified turbulence (Lindborg 2006; Riley and Lindborg 2008), and balanced dynamics near the

Corresponding author: Michael L. Waite, mwaite@uwaterloo.ca tropopause (Tulloch and Smith 2006). Nevertheless, a complete picture of the mesoscale cascade process remains elusive. Indeed, it is possible that more than one mechanism is important, or that different mechanisms act at different levels or scales. In addition to being a fundamental problem in geophysical turbulence, this question has important practical implications: the mesoscale cascade connects the large, energy-containing scales with microscales, where turbulent dissipation occurs, and is not fully resolved by atmospheric models. Parameterizations of the cascade should be consistent with its physics (e.g., Shutts 2005; Schaefer-Rolffs and Becker 2013).

A basic question about the atmospheric energy spectrum, which must be answered before the various proposed theories can be evaluated, is whether it is dominated by IGWs and/or geostrophic vortices. Several studies have attempted to decompose spectra from aircraft data into wave and vortex contributions, mainly using the Helmholtz decomposition of the horizontal velocity field. This decomposition separates the $\mathrm{KE}$ into horizontally rotational and divergent kinetic energy (RKE and DKE, respectively), which are loosely 
attributed to geostrophic vortices and IGWs. This attribution is not exact, even in a linear sense, since IGWs have some rotational motion, especially at low frequencies. Furthermore, at large scales and low latitudes, Rossby waves have some divergent motion. Nonlinearities complicate matters further, since some of the horizontal divergence is balanced, even on an $f$-plane (e.g., Kafiabad and Bartello 2016). Nevertheless, the Helmholtz decomposition is useful and straightforward. Helmholtz-decomposed KE spectra from aircraft data show that, while the KE spectrum is dominated by the RKE in the troposphere (Cho et al. 1999; Lindborg 2007), the level of DKE is not negligible in the troposphere, and actually dominates over the RKE in the lower stratosphere (Callies et al. 2016; Li and Lindborg 2018). Furthermore, Callies et al. (2016) used the Helmholtzdecomposed KE spectra, along with potential energy (PE) spectra from temperature data, to construct linear IGW energy spectra, confirming that wave energy dominates the total mesoscale energy in the lower stratosphere. These studies suggest that the mesoscale spectrum may be explained by IGW theories in the lower stratosphere, but not in the upper troposphere.

Most atmospheric models are capable of reproducing at least the qualitative shape of the atmospheric spectrum when run at sufficiently high resolution (e.g., Koshyk and Hamilton 2001; Skamarock 2004; Hamilton et al. 2008; Skamarock et al. 2014). Such models can be used to address physical questions about the mesoscale cascade. For example, numerical studies have confirmed that the spectral flux is downscale below scales of $100 \mathrm{~km}$ (Augier and Lindborg 2013). Other studies have shown that, while the lower-stratospheric KE spectrum is dominated by DKE consistent with IGWs, the spectral energy budget is strongly influenced by vertical wave fluxes in addition to downscale transfer (Waite and Snyder 2009; Peng et al. 2015a). Nevertheless, there are some differences between models, such as the relatively low fraction of DKE in Hamilton et al. (2008) compared to Skamarock and Klemp (2008). Some of these differences may be due to vertical resolution (Waite 2016; Skamarock et al. 2019) and physical parameterizations (Malardel and Wedi 2016), both of which can affect the simulated spectra.

For spectral analysis from aircraft data, spectra are computed at (approximately) constant height by necessity, and energy decompositions are restricted to horizontal, not vertical, structure. However, analysis of model output allows for more flexibility. An alternative spectral decomposition for model data can be formulated with linear normal modes. The equations of motion can be linearized around a reference state, and solutions to the equations can be expressed as a sum of the orthogonal normal modes of the system (e.g., Kasahara and Puri 1981). By orthogonality, the total domain-averaged energy can be expressed as a sum of modal energies. This approach has several advantages over the usual procedure of analyzing spectra independently at different model levels. First, unlike the Helmholtz decomposition, the normal modes fully separate linear IGWs and geostrophic vortices; there is no ambiguity, at least in a linear sense, about whether the RKE is due to geostrophic vortices or low-frequency IGWs. Second, the vertical structure of the IGWs is built into the decomposition. Third, since potential energy is considered, the normal modes decompose the total mechanical energy, not just the kinetic energy. Finally, since the modes are orthogonal, they allow for a complete decomposition of the total energy into geostrophic vortices and IGWs at every horizontal and vertical scale.

Normal modes have been used to investigate the mesoscale spectrum in both idealized simulations and more realistic model data. Bartello (1995) used normal modes to decompose energy spectra in homogeneous rotating-stratified turbulence, and found that IGW energy cascades downscale with a shallow spectrum due to catalytic wave-vortex interactions. The normal mode approach is now commonly employed in idealized studies of rotating-stratified turbulence (e.g., Kitamura and Matsuda 2010; Deusebio et al. 2013; Herbert et al. 2016). In a more realistic context, Terasaki et al. (2011) and Žagar et al. (2017) used normal modes to decompose the energy spectrum in global reanalysis data. They found that, like Bartello (1995), the IGW spectrum is shallower than the geostrophic spectrum at all scales; indeed, the IGW spectral slope is around $-5 / 3$ across a wide range of length scales. At sufficiently small scales, the shallower IGW spectrum crosses the steeper geostrophic spectrum, and the total energy is dominated by IGWs. In the idealized homogeneous studies, the normal mode structure is Fourier in the horizontal and vertical; by contrast, with global data, the horizontal structure are Hough modes, while the vertical structure is found by solving the associated eigenvalue problem (e.g., Kasahara and Puri 1981).

In this paper, we use normal modes on a periodic $f$-plane to analyze the spectrum in an idealized mesoscale simulation driven by baroclinic instability. Baroclinic waves have been used in several studies for idealized investigations of the mesoscale spectrum (Waite and Snyder 2009; Peng et al. 2015a,b). Despite the simple setup, these simulations yield many realistic mesoscale features, including DKE spectra with a $-5 / 3$ slope. These simulations, while idealized, are able to capture the basic mechanism by which IGWs energy is 
transferred downscale with a $-5 / 3$ spectrum. In dry simulations, the amplitude of the DKE spectrum is not large enough to significantly modify the KE spectrum in the troposphere, but it does in the stratosphere, where the total kinetic energy shallows under the dominance of the DKE (Waite and Snyder 2009). The addition of moist physics (Waite and Snyder 2013; Peng et al. 2015a,b) or topography and surface fluxes (Menchaca and Durran 2019) enhances the DKE spectrum and, as a result, the mesoscale KE spectrum.

Idealized baroclinic instability offers a test case of intermediate complexity in which to investigate the role of IGWs in the mesoscale spectrum. Compared to homogeneous rotating-stratified turbulence, this approach uses more realistic vertical structure and large-scale vortices. On the other hand, the geometry of the $f$-plane allows for the use of Fourier modes to represent the horizontal structure, which are considerably simpler than Hough modes. This setup allows for a careful analysis of the normal mode spectra and comparison between the normal mode and Helmholtz decompositions. The rest of this paper is organized as follows. In section 2, we review the equations for the vertical and horizontal modes. In section 3, we outline the numerical test case and model. Results are presented in section 4 , and conclusions are given in section 5 .

\section{Modes}

\section{a. Separation of variables}

We start by reviewing the derivation of the vertical normal modes for the hydrostatic $f$-plane linearized about a state of rest [we follow Daley (1991)]. The linear adiabatic equations, using pressure as the vertical coordinate, are

$$
\begin{aligned}
\frac{\partial u}{\partial t}-f_{0} v & =-\frac{\partial \phi^{\prime}}{\partial x}, \\
\frac{\partial v}{\partial t}+f_{0} u & =-\frac{\partial \phi^{\prime}}{\partial y}, \\
\nabla \cdot \mathbf{u}+\frac{\partial w}{\partial p} & =0, \\
\frac{\partial}{\partial t} \frac{\partial \tilde{\phi}}{\partial p}+w \tilde{\Gamma} & =0,
\end{aligned}
$$

where $(u, v)$ is the horizontal velocity, $w=\dot{p}$ is the vertical velocity (the dot denotes a time derivative; we use $\omega$ for frequency below), $p$ is the pressure, $f_{0}$ is the Coriolis parameter, $\tilde{\phi}$ and $\phi^{\prime}$ are the basic state and perturbation geopotential, and $\tilde{\Gamma}$ is the static stability, given by

$$
\tilde{\Gamma}(p)=\frac{1}{p} \frac{d}{d p}\left(p \frac{d \tilde{\phi}}{d p}-\frac{R \tilde{\phi}}{c_{p}}\right) .
$$

By assuming separable dependence on $(x, y, t)$ and $p$, the variables can be expressed as

$$
\begin{aligned}
u(x, y, p, t) & =U(x, y, t) Z(p), \\
v(x, y, p, t) & =V(x, y, t) Z(p), \\
\phi^{\prime}(x, y, p, t) & =\Phi(x, y, t) Z(p) .
\end{aligned}
$$

Equations for the time-varying horizontal structure $U(x, y, t), V(x, y, t)$, and $\Phi(x, y, t)$ and the fixed vertical structure $Z(p)$ are then

$$
\begin{aligned}
\frac{\partial U}{\partial t}-f_{0} V & =-\frac{\partial \Phi}{\partial x}, \\
\frac{\partial V}{\partial t}+f_{0} U & =-\frac{\partial \Phi}{\partial y}, \\
\frac{1}{Z} \frac{d}{d p}\left(\frac{1}{\tilde{\Gamma}} \frac{d Z}{d p}\right) & =\frac{1}{\partial \Phi / \partial t}\left(\frac{\partial U}{\partial x}+\frac{\partial V}{\partial y}\right)=-\frac{1}{g h},
\end{aligned}
$$

where $g$ is gravity and $-1 /(g h)$ is the separation constant in the mixed Eq. (11), which defines the equivalent depth $h$.

The equations of motion are now in a separated state consisting of the shallow-water equations of depth $h$ in the horizontal,

$$
\begin{aligned}
\frac{\partial U}{\partial t}-f_{0} V & =-\frac{\partial \Phi}{\partial x}, \\
\frac{\partial V}{\partial t}+f_{0} U & =-\frac{\partial \Phi}{\partial y}, \\
\frac{\partial \Phi}{\partial t}+g h\left(\frac{\partial U}{\partial x}+\frac{\partial V}{\partial y}\right) & =0
\end{aligned}
$$

and the vertical structure equation,

$$
\frac{d}{d p}\left(\frac{1}{\tilde{\Gamma}} \frac{d Z}{d p}\right)+\frac{1}{g h} Z=0 .
$$

When the divergence is not identically zero, the horizontal equations are influenced by the vertical structure through the value of $1 /(g h)$, which is an eigenvalue of Eq. (15). Because they are based on the linear equations, the horizontal and vertical Eqs. (12)-(15) have the same form when different vertical coordinates, for example, terrain-following sigma coordinates, are used (Staniforth et al. 1985).

\section{b. Vertical modes}

The vertical structure Eq. (15) is an eigenvalue problem, which can be solved with rigid boundary 
conditions $\dot{z}=0$, where $z$ is the height, at the upper and lower boundaries:

$$
\begin{aligned}
& \frac{d Z}{d p}+\frac{p_{s} \tilde{\Gamma}}{R T_{s}} Z=0 \quad \text { at } \quad p=p_{s}, \\
& \frac{d Z}{d p}+\frac{p_{t} \tilde{\Gamma}}{R T_{t}} Z=0 \quad \text { at } \quad p=p_{t}
\end{aligned}
$$

where subscripts $s$ and $t$ denote the surface and model top, respectively (Daley 1991). Rigid-lid boundary conditions, while not exactly realistic at the upper boundary, are convenient, consistent with model boundary conditions, and commonly employed (e.g., Wiin-Nielsen 1971; Kasahara and Qian 2000). Cohn and Dee (1989) point out that the spectrum of vertical modes is determined by the coefficients of Eq. (15) near the upper boundary. Together, Eqs. (15)-(17) are a standard Sturm-Liouville problem and define the vertical structure. Solutions are given by a sequence of vertical normal modes $Z_{n}(p)$ for $n=0,1,2, \ldots$, which are orthogonal in the $L^{2}$ norm in $p$, and eigenvalues $1 /\left(g h_{n}\right)$. Eigenvalues define the equivalent depth $h_{n}$ of the $n$th mode.

\section{c. Horizontal modes}

Once the vertical structure problem is solved, the equivalent depths $h_{n}$ fully determine the horizontal problem in Eqs. (12)-(14). These equations are the linear shallow-water equations with depth $h_{n}$, the normal modes of which have been extensively studied on the sphere (e.g., Kasahara 1976). The horizontally periodic $f$-plane is considerably simpler because a Fourier basis can be used [we follow Warn (1986)]. Assuming harmonic time dependence, $U, V$, and $\Phi \propto \exp (-i \omega t)$, where $i$ is the imaginary unit, and Eqs. (12)-(14) become

$$
\begin{gathered}
-i \omega U_{n}-f_{0} V_{n}+\frac{\partial \Phi_{n}}{\partial x}=0, \\
-i \omega V_{n}+f_{0} U_{n}+\frac{\partial \Phi_{n}}{\partial y}=0, \\
\frac{\partial U_{n}}{\partial x}+\frac{\partial V_{n}}{\partial y}-\frac{i \omega}{g h_{n}} \Phi_{n}=0,
\end{gathered}
$$

where subscript $n$ denotes horizontal variables corresponding to vertical mode $n$. The corresponding Fourier coefficients satisfy

$$
\begin{gathered}
-i \omega \hat{U}_{n}-f_{0} \hat{V}_{n}+i k_{x} \hat{\Phi}_{n}=0, \\
-i \omega \hat{V}_{n}+f_{0} \hat{U}_{n}+i k_{y} \hat{\Phi}_{n}=0, \\
i k_{x} \hat{U}_{n}+i k_{y} \hat{V}_{n}-\frac{i \omega}{g h_{n}} \hat{\Phi}_{n}=0,
\end{gathered}
$$

where $\hat{f}_{n}(\mathbf{k})$ is the Fourier coefficient of the field $f_{n}(x, y)$ and $\mathbf{k}=\left(k_{x}, k_{y}\right)$ is the horizontal wave vector. This results in a $3 \times 3$ eigenvalue problem at every $(\mathbf{k}, n)$, given by

$$
\omega\left(\begin{array}{l}
\hat{U}_{n} \\
\hat{V}_{n} \\
\hat{\eta}_{n}
\end{array}\right)=\left(\begin{array}{ccc}
0 & i f_{0} & k_{x} c_{n} \\
-i f_{0} & 0 & k_{y} c_{n} \\
k_{x} c_{n} & k_{y} c_{n} & 0
\end{array}\right)\left(\begin{array}{c}
\hat{U}_{n} \\
\hat{V}_{n} \\
\hat{\eta}_{n}
\end{array}\right),
$$

where $c_{n}=\sqrt{g h_{n}}$ is the gravity wave speed for vertical mode $n$ and $\eta_{n}=\Phi_{n} / c_{n}$ is the scaled geopotential, which has units of velocity.

The system (24) has eigenvalues

$$
\omega_{\mathbf{k}, n}^{0}=0, \quad \omega_{\mathbf{k}, n}^{ \pm}= \pm \sqrt{c_{n}^{2} k^{2}+f_{0}^{2}},
$$

and corresponding orthogonal eigenvectors

$$
\begin{aligned}
& \mathbf{E}_{\mathbf{k}, n}^{0}=\frac{1}{\sqrt{c_{n}^{2} k^{2}+f_{0}^{2}}}\left(\begin{array}{c}
-i c_{n} k_{y} \\
i c_{n} k_{x} \\
f_{0}
\end{array}\right) \\
& \mathbf{E}_{\mathbf{k}, n}^{ \pm}=\frac{1}{\sqrt{2 k} \sqrt{c_{n}^{2} k^{2}+f_{0}^{2}}}\left(\begin{array}{c}
i f_{0} k_{y} \pm k_{x} \sqrt{c_{n}^{2} k^{2}+f_{0}^{2}} \\
-i f_{0} k_{x} \pm k_{y} \sqrt{c_{n}^{2} k^{2}+f_{0}^{2}} \\
c_{n} k^{2}
\end{array}\right)
\end{aligned}
$$

where $k^{2}=k_{x}^{2}+k_{y}^{2}$ [our eigenvector notation follows Bartello (1995), who considered the nonhydrostatic uniformly stratified case]. For every vertical mode $n$ and horizontal wave vector $\mathbf{k}$, there are three horizontal modes: one geostrophic mode with frequency $\omega_{\mathbf{k}, n}^{0}$, which yields steady geostrophic motion; and two ageostrophic modes with frequencies $\omega_{\mathbf{k}, n}^{ \pm}$, which correspond to left- and right-traveling inertia-gravity waves. The Rossby deformation wavenumber $k_{n}^{R}=f_{0} / c_{n}$, which is determined by $c_{n}$ and therefore by the equivalent depth $h_{n}$, separates large rotation-dominated scales $\left(k \ll k_{n}^{R}\right)$, at which inertia-gravity waves are approximately inertia waves $\left(\omega_{\mathbf{k}, n}^{ \pm} \approx \pm f_{0}\right)$, from small gravity-dominated scales $\left(k \gg k_{n}^{R}\right)$, at which waves are approximately gravity waves $\left(\omega_{\mathbf{k}, n}^{ \pm} \approx \pm c_{n} k\right)$.

For a given $(\mathbf{k}, n)$, the Fourier coefficients of the velocity and scaled geopotential can be expressed as a linear combination of the geostrophic and ageostrophic eigenvectors

$$
\left(\begin{array}{c}
\hat{U}_{n} \\
\hat{V}_{n} \\
\hat{\eta}_{n}
\end{array}\right)=A_{\mathbf{k}, n}^{0} \mathbf{E}_{\mathbf{k}, n}^{0}+A_{\mathbf{k}, n}^{+} \mathbf{E}_{\mathbf{k}, n}^{+}+A_{\mathbf{k}, n}^{-} \mathbf{E}_{\mathbf{k}, n}^{-},
$$


where $A_{\mathbf{k}, n}^{0}$ and $A_{\mathbf{k}, n}^{ \pm}$are the amplitudes of the geostrophic and ageostrophic components. These amplitudes are found by taking the inner product of Eq. (28) with the eigenvectors; they are given by

$$
A_{\mathbf{k}, n}^{0}=\left(\begin{array}{c}
\hat{U}_{n} \\
\hat{V}_{n} \\
\hat{\eta}_{n}
\end{array}\right) \cdot \mathbf{E}_{\mathbf{k}, n}^{0}=\frac{c_{n} \hat{\zeta}_{n}+f_{0} \hat{\eta}_{n}}{\sqrt{c_{n}^{2} k^{2}+f_{0}^{2}}}
$$

and similarly

$$
A_{\mathbf{k}, n}^{ \pm}=\left(\begin{array}{c}
\hat{U}_{n} \\
\hat{V}_{n} \\
\hat{\eta}_{n}
\end{array}\right) \cdot \mathbf{E}_{\mathbf{k}, n}^{ \pm}=\frac{-f_{0} \hat{\zeta}_{n} \mp i \sqrt{c_{n}^{2} k^{2}+f_{0}^{2}} \hat{\delta}_{n}+c_{n} k^{2} \hat{\eta}_{n}}{\sqrt{2 k} \sqrt{c_{n}^{2} k^{2}+f_{0}^{2}}}
$$

where $\hat{\zeta}_{n}=i k_{x} \hat{V}_{n}-i k_{y} \hat{U}_{n}$ and $\hat{\delta}_{n}=i k_{x} \hat{U}_{n}+i k_{y} \hat{V}_{n}$ are the vertical vorticity and horizontal divergence of mode $n$.

In summary, the full three-dimensional dynamical fields can decomposed, first into vertical modes, and then into horizontal modes. For each vertical mode $n$ and each horizontal wave vector $\mathbf{k}$, there are three modes: one geostrophic mode (with amplitude $A_{\mathbf{k}, n}^{0}$ ) and two ageostrophic modes (with amplitudes $A_{\mathbf{k}, n}^{ \pm}$). A similar decomposition into geostrophic (Rossby wave) and ageostrophic (inertiagravity wave) modes, using Hough instead of Fourier modes to describe the horizontal structure, is possible on the sphere (Kasahara 1976). By the dispersion relation (25), the ageostrophic modes are equivalent to linear inertia-gravity waves, as documented in many analogous contexts [e.g., Warn (1986) in shallow water; Bartello (1995) in homogeneous rotating-stratified turbulence; and Žagar et al. (2017) on the sphere]. As a linear decomposition, the geostrophic and ageostrophic modes do not directly separate nonlinearly balanced vortices and unbalanced waves, respectively; for example, balanced vortices will project somewhat on to the ageostrophic modes (Kafiabad and Bartello 2016).

\section{d. Reconstruction and energetics}

Since the vertical modes $Z_{n}(p)$ are orthogonal and complete, we can use them to express three-dimensional velocity and geopotential fields $f(x, y, p)$ as

$$
f(x, y, p)=\sum_{n} f_{n}(x, y) Z_{n}(p),
$$

where the $f_{n}$ can be found by projection onto $Z_{n}(p)$ as

$$
f_{n}(x, y)=\frac{1}{p_{s}-p_{t}} \int_{p_{t}}^{p_{s}} f(x, y, p) Z_{n}(p) d p
$$

Furthermore, with periodic boundary conditions in the horizontal, $f(x, y, p)$ can be expressed in terms of vertical modes and horizontal Fourier modes as

$$
f(x, y, p)=\sum_{\mathbf{k}, n} \hat{f}_{n}(\mathbf{k}) e^{i\left(k_{x} x+k_{y} y\right)} Z_{n}(p) .
$$

Parseval's theorem relates the domain-averaged square of $f$ (e.g., the zonal kinetic energy for $f=u$ ) to the sum of the modal coefficients:

$$
\frac{1}{A\left(p_{s}-p_{t}\right)} \iiint_{V} f^{2}(x, y, p) d x d y d p=\sum_{\mathbf{k}, n}\left|\hat{f}_{n}(\mathbf{k})\right|^{2},
$$

where $A$ is the horizontal area of the domain. Note that, using velocity for $f$, the RHS of Eq. (34) is proportional to the kinetic energy per unit volume, not mass, because the vertical integral is over $p$; no extra factor of density is required. For a given vertical mode $n$, the total energy can be decomposed into kinetic energy (KE) and potential energy (PE) as

$$
\begin{aligned}
E_{n} & =\sum_{\mathbf{k}} \underbrace{\frac{1}{2}\left(\left|\hat{U}_{n}(\mathbf{k})\right|^{2}+\left|\hat{V}_{n}(\mathbf{k})\right|^{2}\right)}_{\text {Kinetic }}+\underbrace{\frac{1}{2}\left|\hat{\eta}_{n}(\mathbf{k})\right|^{2}}_{\text {Potential }} \\
& =\sum_{\mathbf{k}} \operatorname{KE}(\mathbf{k}, n)+\operatorname{PE}(\mathbf{k}, n),
\end{aligned}
$$

and into geostrophic energy (GE) and ageostrophic energy (AE) as

$$
\begin{aligned}
E_{n} & =\sum_{\mathbf{k}} \underbrace{\frac{1}{2}\left|A_{\mathbf{k}, n}^{0}\right|^{2}}_{\text {Geostrophic }}+\underbrace{\frac{1}{2}\left(\left|A_{\mathbf{k}, n}^{+}\right|^{2}+\left|A_{\mathbf{k}, n}^{-}\right|^{2}\right)}_{\text {Ageostrophic }} \\
& =\sum_{\mathbf{k}} \operatorname{GE}(\mathbf{k}, n)+\operatorname{AE}(\mathbf{k}, n) .
\end{aligned}
$$

Furthermore, for $k>0$ the kinetic energy spectrum for each vertical mode can be decomposed using Helmholtz decomposition into rotational kinetic energy and divergent kinetic energy

$$
\begin{aligned}
\operatorname{KE}(\mathbf{k}, n) & =\frac{1}{2} \frac{\left|\hat{\zeta}_{n}(\mathbf{k})\right|^{2}}{k^{2}}+\frac{1}{2} \frac{\left|\hat{\delta}_{n}(\mathbf{k})\right|^{2}}{k^{2}} \\
& =\operatorname{RKE}(\mathbf{k}, n)+\operatorname{DKE}(\mathbf{k}, n) .
\end{aligned}
$$

From these modal spectra, horizontal wavenumber spectra can be computed by summing over annuli in the $k_{x}-k_{y}$ plane (e.g., Waite and Snyder 2009).

\section{e. Relationship between modal and Helmholtz decomposition}

The horizontal modal amplitudes in Eqs. (29) and (30) show that there is a relationship between the normal mode and Helmholtz decompositions: the geostrophic mode in Eq. (29) depends on the horizontal vorticity but not the divergence, while the ageostrophic mode in Eq. (30) depends on vorticity and divergence, as expected on an $f$-plane (on a $\beta$-plane or sphere, the 
geostrophic mode additionally has some divergence due to the meridional dependence of the Coriolis parameter, but this is small at midlatitude mesoscales). We explore this connection further here. First, consider the relationship between the geostrophic and rotational kinetic energy spectra. It can be shown that for large wavenumbers with $k \gg k_{n}^{R}$, the geostrophic energy spectrum satisfies

$$
\mathrm{GE}(\mathbf{k}, n) \approx \operatorname{RKE}(\mathbf{k}, n) .
$$

To derive this approximation, consider the size of the geostrophic modal amplitude from Eq. (29)

$$
\begin{aligned}
\left|A_{\mathbf{k}, n}^{0}\right|^{2}= & \frac{1}{1+\left(f_{0} / c_{n} k\right)^{2}}\left[\frac{\left|\hat{\zeta}_{n}\right|^{2}}{k^{2}}+\left(\frac{f_{0}}{c_{n} k}\right)^{2}\left|\hat{\eta}_{n}\right|^{2}\right. \\
& \left.+\frac{f_{0}}{c_{n} k}\left(\hat{\eta}_{n}^{*} \frac{\hat{\zeta}_{n}}{k}+\text { c.c. }\right)\right]
\end{aligned}
$$

where c.c. denotes complex conjugate. Therefore, the geostrophic energy is

$$
\begin{aligned}
\operatorname{GE}(\mathbf{k}, n)= & \frac{1}{2}\left|A_{\mathbf{k}, n}^{0}\right|^{2} \\
= & \frac{1}{1+\left(k_{n}^{R} / k\right)^{2}}\left[\operatorname{RKE}(\mathbf{k}, n)+\left(\frac{k_{n}^{R}}{k}\right)^{2} \operatorname{PE}(\mathbf{k}, n)\right. \\
& \left.+\frac{k_{n}^{R}}{2 k}\left(\hat{\eta}_{n}^{*} \frac{\hat{\zeta}_{n}}{k}+\text { c.c. }\right)\right]
\end{aligned}
$$

The third term is bounded by

$$
\begin{aligned}
& \mid \frac{k_{n}^{R}}{2 k} \frac{1}{1+\left(k_{n}^{R} / k\right)^{2}}\left(\frac{\hat{\eta}_{n}^{*} \hat{\zeta}_{n}}{k}+\text { c.c. }\right) \mid \\
& \quad \leq 2 \frac{k_{n}^{R}}{k} \frac{1}{1+\left(k_{n}^{R} / k\right)^{2}} \sqrt{\operatorname{RKE}(\mathbf{k}, n) \operatorname{PE}(\mathbf{k}, n)} .
\end{aligned}
$$

This approximation requires that the potential energy is not much larger than the rotational kinetic energy when $k \gg k_{n}^{R}$, which will be investigated below. If it is, then a larger wavenumber than $k_{n}^{R}$ may be required for the potential energy contribution to the GE to be negligible. For $k \gg k_{n}^{R}$, Eq. (40) becomes Eq. (38). Therefore, at scales much smaller than the deformation scale, the geostrophic energy is approximately equal to the rotational kinetic energy. As the ratio $k_{n}^{R} / k$ increases, for example, by decreasing $k$ at fixed $n$ or by increasing $n$ at fixed $k$, the geostrophic energy has a reduced contribution from the rotational kinetic energy and additional contribution from the potential energy. Similarly, for small wavenumbers with $k \ll k_{n}^{R}$, we have

$$
\mathrm{GE}(\mathbf{k}, n) \approx \operatorname{PE}(\mathbf{k}, n),
$$

and the large-scale geostrophic energy is approximately equal to the potential energy.

Next, consider the relationship between the ageostrophic and divergent kinetic energy. For $k \gg k_{n}^{R}$, the ageostrophic energy spectrum satisfies

$$
\operatorname{AE}(\mathbf{k}, n) \approx \operatorname{DKE}(\mathbf{k}, n)+\operatorname{PE}(\mathbf{k}, n) .
$$

To derive this approximation, consider the size of the ageostrophic modal amplitudes from Eq. (30)

$$
\begin{aligned}
\left|A_{\mathbf{k}, n}^{ \pm}\right|^{2}= & \frac{1}{2} \frac{1}{1+\left(k c_{n} / f_{0}\right)^{2}} \frac{\left|\hat{\zeta}_{n}\right|^{2}}{k^{2}}+\frac{1}{2} \frac{\left|\hat{\delta}_{n}\right|^{2}}{k^{2}}+\frac{1}{2} \frac{1}{1+\left[f_{0} /\left(c_{n} k\right)\right]^{2}}\left|\hat{\eta}_{n}\right|^{2} \\
& +\left[ \pm \frac{i\left(f_{0} / c_{n}\right)}{2 k^{2} \sqrt{k^{2}+\left(f_{0} / c_{n}\right)^{2}}} \hat{\zeta}_{n}^{*} \hat{\delta}_{n} \pm \frac{i}{2 \sqrt{k^{2}+\left(f_{0} / c_{n}\right)^{2}}} \hat{\delta}_{n}^{*} \hat{\eta}_{n}-\frac{f_{0} / c_{n}}{2\left[k^{2}+\left(f_{0} / c_{n}\right)^{2}\right]^{3}} \hat{\zeta}_{n}^{*} \hat{\eta}_{n}+\text { c.c. }\right] .
\end{aligned}
$$

When the + and - modes are added together to construct the total ageostrophic energy, the first two cross terms on the RHS cancel, and we are left with

$$
\begin{aligned}
\operatorname{AE}(\mathbf{k}, n)= & \frac{1}{2}\left(\left|A_{\mathbf{k}, n}^{+}\right|^{2}+\left|A_{\mathbf{k}, n}^{-}\right|^{2}\right) \\
= & \frac{1}{1+\left(k / k_{n}^{R}\right)^{2}} \operatorname{RKE}(\mathbf{k}, n)+\operatorname{DKE}(\mathbf{k}, n)+\frac{1}{1+\left(k_{n}^{R} / k\right)^{2}} \operatorname{PE}(\mathbf{k}, n) \\
& -\frac{k_{n}^{R}}{2 k} \frac{1}{1+\left(k_{n}^{R} / k\right)^{2}}\left(\frac{\hat{\zeta}_{n}^{*}}{k} \hat{\eta}_{n}+\text { c.c. }\right) .
\end{aligned}
$$


The last term is bounded by Eq. (41), as in the geostrophic spectrum. For $k \gg k_{n}^{R}$, this becomes Eq. (43). This approximation assumes that the RKE is not much larger than the DKE and PE when $k \gg k_{n}^{R}$, which will be checked below. Therefore, at scales much smaller than the deformation scale, the ageostrophic energy is the sum of the DKE and PE. As the ratio $k_{n}^{R} / k$ increases, the contribution from the DKE remains the same, but the RKE contribution increases and the PE contribution decreases. Similarly, for $k \ll k_{n}^{R}$, we have

$\operatorname{AE}(\mathbf{k}, n) \approx \operatorname{RKE}(\mathbf{k}, n)+\operatorname{DKE}(\mathbf{k}, n)=\operatorname{KE}(\mathbf{k}, n)$,

and the large-scale ageostrophic energy approximately equals the kinetic energy.

This analysis suggests that, for sufficiently small horizontal scales with $k \gg k_{n}^{R}$, the Helmholtz decomposition does give a good approximation to the geostrophic/ageostrophic decomposition. This agreement will be checked below. At larger scales, the relationship between the two decompositions is different. Furthermore, in a $\beta$-plane or spherical model, the relationship between the two decompositions would be complicated by the presence of velocity divergence in the geostrophic (Rossby wave) mode.

\section{Numerical approach}

\section{a. Initial conditions}

We initialize our simulations with a baroclinically unstable double jet based on the idealized channel jet of Ullrich et al. (2015). The velocity of a single zonal jet, with width $\ell$, is given by

$$
u(x, y, \sigma)=-u_{0} \sin ^{2}\left(\frac{\pi y}{\ell}\right) \ln \sigma \exp \left[-\left(\frac{\ln \sigma}{b}\right)^{2}\right],
$$

where $\sigma=p / p_{s}$ and $u_{0}$ and $b$ are the velocity and depth scale of the jet. These and other basic parameters are given in Table 1 . The prescribed zonal velocity field goes to zero at $y=0, \ell$. With $b=2$, the velocity reaches a maximum value of $0.86 u_{0}$ at a height of $\sigma=$ 0.24 . The initial Rossby number, based on the jet parameters in Table 1 , is $\mathrm{Ro}_{0}=u_{0} /\left(f_{0} \ell\right)=0.21$.

The geopotential field has a basic state and fluctuation

$$
\phi(x, y, \sigma)=\tilde{\phi}(\sigma)+\phi^{\prime \prime}(x, y) \ln \sigma \exp \left[-\left(\frac{\ln \sigma}{b}\right)^{2}\right],
$$

where the basic-state profile is
TABLE 1. Parameters used in the initialization and simulation.

\begin{tabular}{clc}
\hline \hline Parameter & \multicolumn{1}{c}{ Description } & Value \\
\hline$u_{0}$ & Velocity scale & $55 \mathrm{~m} \mathrm{~s}^{-1}$ \\
$\ell$ & Single jet width & $2560 \mathrm{~km}$ \\
$b$ & Dimensionless jet depth & 2 \\
$T_{0}$ & Surface temperature & $288 \mathrm{~K}$ \\
$g$ & Acceleration due to gravity & $9.81 \mathrm{~m} \mathrm{~s}^{-1}$ \\
$\Gamma$ & Lapse rate & $0.005 \mathrm{~K} \mathrm{~m}^{-1}$ \\
$R_{d}$ & Ideal gas constant of dry air & $287 \mathrm{~J} \mathrm{~kg}^{-1} \mathrm{~K}^{-1}$ \\
$f_{0}$ & Coriolis parameter & $10^{-4} \mathrm{~s}^{-1}$ \\
$L$ & Domain size & $5120 \mathrm{~km}$ \\
$\Delta x=\Delta y$ & Grid spacing & $5 \mathrm{~km}$ \\
$p_{s}$ & Surface pressure (basic state) & $1017.5 \mathrm{hPa}$ \\
$p_{t}$ & Lid pressure (basic state) & $7.0 \mathrm{hPa}$ \\
\hline
\end{tabular}

$$
\tilde{\phi}(\sigma)=\frac{T_{0} g}{\Gamma}\left(1-\sigma^{\frac{R_{d} \Gamma}{g}}\right)
$$

and the horizontal structure of the fluctuation is

$$
\phi^{\prime \prime}(x, y)=\frac{u_{0} f_{0}}{2}\left[y-\frac{\ell}{2}-\frac{\ell}{2 \pi} \sin \left(\frac{2 \pi y}{\ell}\right)\right] .
$$

Here $T_{0}$ is the surface temperature, $g$ is the acceleration due to gravity, $R_{d}$ is the ideal gas constant for dry air, and $\Gamma$ is the (constant) lapse rate of the atmosphere. Note that this $\Gamma$ is different from Daley's (1991) static stability profile $\tilde{\Gamma}(p)$ from section 2 .

In addition to prescribing the velocity fields and geopotential field, a temperature field is required to completely describe the initial state of the jet. The basicstate temperature is given by

$$
\tilde{T}(\sigma)=T_{0} \sigma^{\frac{R_{d} \Gamma}{g}}
$$

which satisfies $d \tilde{T} / d z=-\Gamma$. The fluctuation temperature $T^{\prime}(x, y, \sigma)=T(x, y, \sigma)-\tilde{T}(\sigma)$ is

$$
T^{\prime}(x, y, \sigma)=\frac{\phi^{\prime \prime}(x, y)}{R_{d}}\left[\frac{2}{b^{2}}(\ln \sigma)^{2}-1\right] \exp \left[-\left(\frac{\ln \sigma}{b}\right)^{2}\right] \text {. }
$$

Figure 1 shows the basic-state temperature and Brunt-Väisälä frequency. Although the initial jet lacks a tropopause, the atmosphere is still stably stratified with a sharp increase in stratification above $100 \mathrm{hPa}$.

To use periodic horizontal boundary conditions, which simplifies the analysis of the horizontal normal modes, we extend the jet meridionally to construct a doubly periodic double jet. The domain is extended from $y \in[0, \ell]$ to $y \in[-\ell, \ell]$, so the full domain width is $L=2 \ell$. The geopotential and temperature fields are chosen to be even extensions about $y=0$, and the zonal 

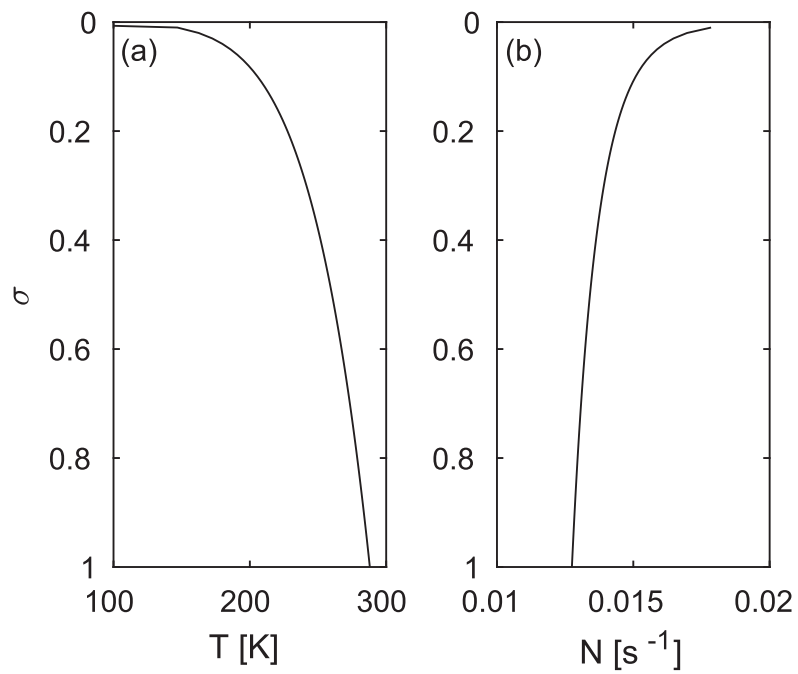

FIG. 1. Initial mean vertical profile of the (a) horizontal mean temperature and (b) Brunt-Väisälä frequency.

velocity field is an odd extension to maintain geostrophic balance. Figure 2 shows the zonal velocity and potential temperature fields in the extended domain.

Finally, to initialize baroclinic instability, each jet is perturbed with a two-dimensional Gaussian potential temperature bubble of amplitude $4 \mathrm{~K}$ and half-width $600 \mathrm{~km}$ (Ullrich et al. 2015) centered at $y= \pm \ell / 2$. The geopotential and density are recalculated after the perturbation to maintain column mass and hydrostatic balance.

\section{b. Model setup}

Simulations are performed with the Advanced Research dynamical core of the Weather Research and Forecasting (WRF, version 3.7.1) Model (Skamarock et al. 2008), which solves the equations for a compressible, nonhydrostatic atmosphere. The domain is a square doubly periodic $f$-plane with size $L \times L$. The horizontal grid spacing is $\Delta x=\Delta y=5 \mathrm{~km}$. The domain depth is $25 \mathrm{~km}$, with 100 levels evenly spaced in the basic-state pressure. The grid spacing $\Delta z$ therefore increases with height from $84 \mathrm{~m}$ near the surface, to $260 \mathrm{~m}$ at $z=10 \mathrm{~km}$, to $2 \mathrm{~km}$ near the top. The basic-state pressure at the model surface and lid are $p_{s}=1017.5 \mathrm{hPa}$ and $p_{t}=$ $7.0 \mathrm{hPa}$. The time step is $\Delta t=30 \mathrm{~s}$.

Since these are idealized simulations, most physical parameterizations are turned off. Simulations are dry and there are no surface fluxes, radiative transfer, or boundary layer mixing. A diffusive damping layer with dimensionless coefficient 0.05 is employed within $5 \mathrm{~km}$ of the upper boundary. The advection scheme is fifth order in the horizontal and third order in the vertical;

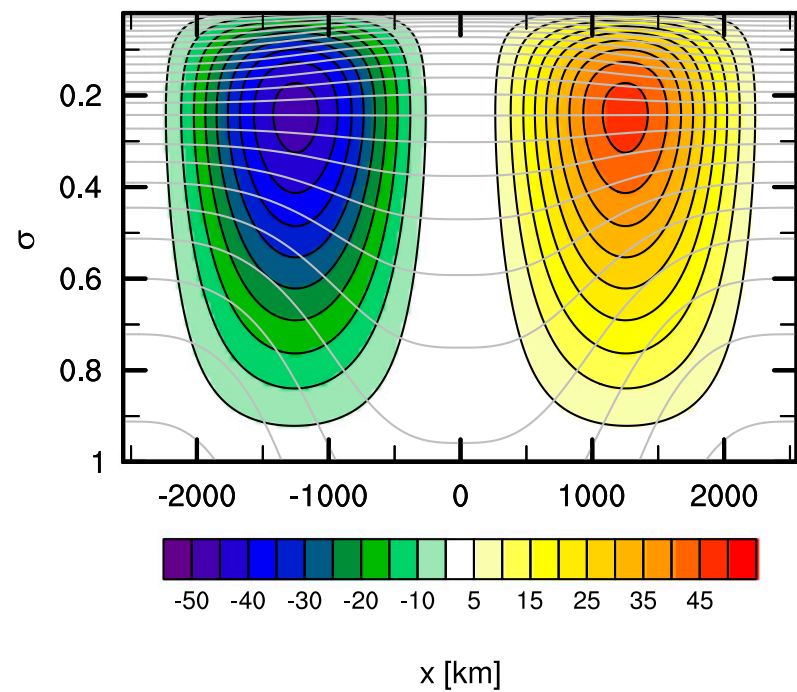

FIG. 2. Meridional slice of initial velocity (colored; $\mathrm{m} \mathrm{s}^{-1}$ ) and potential temperature (gray contours; interval: $10 \mathrm{~K}$ ) after the domain has been extended to be doubly periodic.

the weak numerical viscosity from the upwind-biased fluxes is sufficient to suppress gridscale noise, and no additional eddy viscosity is imposed (e.g., Wicker and Skamarock 2002). Simulations were run for 16 days with fields output every $2 \mathrm{~h}$.

\section{c. Vertical modes}

To analyze the simulation output in terms of the normal modes, we need the set of vertical modes $Z_{n}(p)$ and equivalent depths $h_{n}$ associated with the background state and numerical grid of our simulation. We compute the modes from the initial basic-state $\tilde{\phi}$ in Eq. (49), which, through the static stability $\tilde{\Gamma}$, determines the vertical structure in Eq. (15). Although the model is nonhydrostatic, dynamics at the scales of interest are close to hydrostatic, so the hydrostatic normal modes are used. The eigenvalue problem is discretized using a second-order centered finite difference scheme with evenly spaced pressure levels (Daley 1991), which approximates the modes $Z_{n}(p)$ at the WRF mass points. Finite difference solutions to the eigenvalue problem have been successfully employed in other numerical studies of vertical modes in atmospheric models (e.g., Kasahara and Puri 1981; Tanaka 1985). Žagar et al. (2012) analyzed ECMWF model output using vertical modes discretized with both finite difference and higherorder spectral approximations; the resulting projections and energetics were not significantly affected by the discretization approach. The eigenvalue solver was verified by comparison with the isothermal modes, for which there is an analytical solution (e.g., Daley 1991). Since the WRF Model levels are equally spaced in basic 
state pressure, no vertical interpolation is employed, either in the calculation of the modes or the projection onto them.

Figure 3 shows the computed equivalent depths $h_{n}$ and deformation wavenumber $k_{n}^{R}$ (here and elsewhere, wavenumbers are nondimensionalized by $\Delta k=2 \pi / L$ ). With 100 model levels, there are 100 orthogonal vertical modes: a barotropic mode $n=0$ and baroclinic modes $1 \leq n \leq 99$. For clarity, Figs. $3 \mathrm{a}$ and $3 \mathrm{~b}$ are restricted to $0 \leq n \leq 30$, while Figs. $3 \mathrm{c}$ and $3 \mathrm{~d}$ show the full range of $n$. The equivalent depth of the barotropic mode is $9753 \mathrm{~m}$, and the depths of the first few baroclinic modes are $1290,448,215$, and $125 \mathrm{~m}$. The dimensionless deformation wavenumbers range from 0.7 for $n=1$ to 6 for $n=10$, to 13 for $n=20$, and to 31 for $n=40$. Figure 4 shows the vertical structure of a selection of modes. Mode $n$ has $n$ zero crossings, as expected. The distance between two consecutive zero crossings around $\sigma=0.5$, that is, half the approximate modal wavelength in the midtroposphere, decreases from $6.5 \mathrm{~km}$ for $n=5$ to $3.2 \mathrm{~km}, 1.4 \mathrm{~km}$, and $900 \mathrm{~m}$ for $n=10,20$, and 30 , respectively. The wavelength decreases for increasing $n$, and around approximately $n=20$, the modes become marginally resolved near the upper part of the domain. For higher modes, the oscillations cease in the upper portion of the domain, which extends downward with increasing $n$. Terasaki and Tanaka (2007) found that the lack of oscillations at upper levels for higher modes is due to the finite difference method, and occurs when there are very high-frequency oscillations in the analytical modes. Žagar et al. (2012) found small-amplitude high-frequency oscillations at these levels when higher-order discretizations were used, but they did not significantly affect the results. Even when the modes are not well resolved, they form an orthogonal basis.

\section{Results}

\section{a. Simulation overview}

Figure 5 shows the evolution of the potential temperature in the lowest grid cell and $500-\mathrm{hPa}$ vertical vorticity and horizontal divergence at $t=0,4,8,12$, and 16 days. At the initial time, the jet structure and temperature bubble are clearly visible; since the initial jet is in geostrophic balance, there is no velocity divergence. By 4 days, a single baroclinic wave is growing on each jet, accompanied by a small band of inertia-gravity waves in the divergence field. By 8 days, two mature baroclinic waves are present on each jet, with clearly defined cold regions, cyclonic vorticity, and embedded inertia-gravity waves. Waves are visible above the cold fronts and inside the cyclones. By 12 days and beyond,
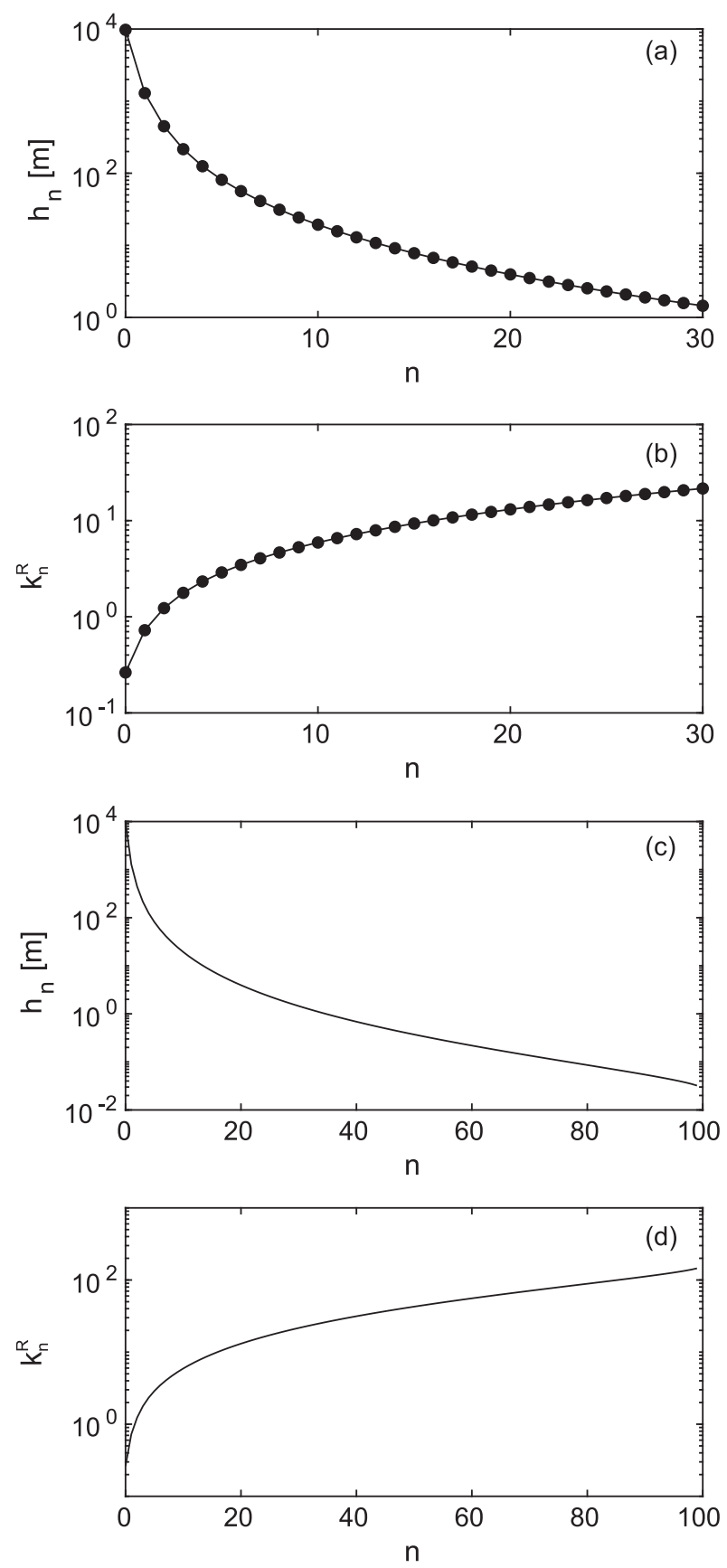

FIG. 3. (a),(c) Equivalent depths $h_{n}$ and (b),(d) deformation wavenumbers $k_{n}^{R}$ for (a),(b) the first 30 and (c),(d) all vertical modes. In (b) and (d), $k_{n}^{R}$ is nondimensionalized by $2 \pi / L_{x}$.

the baroclinic instability appears to have saturated, instabilities are developing on the surface potential temperature filaments, and waves fill the entire domain.

\section{b. Vertically averaged kinetic energy spectra}

Figure 6 shows vertically averaged spectra of kinetic, rotational kinetic, and divergent kinetic energy at a 

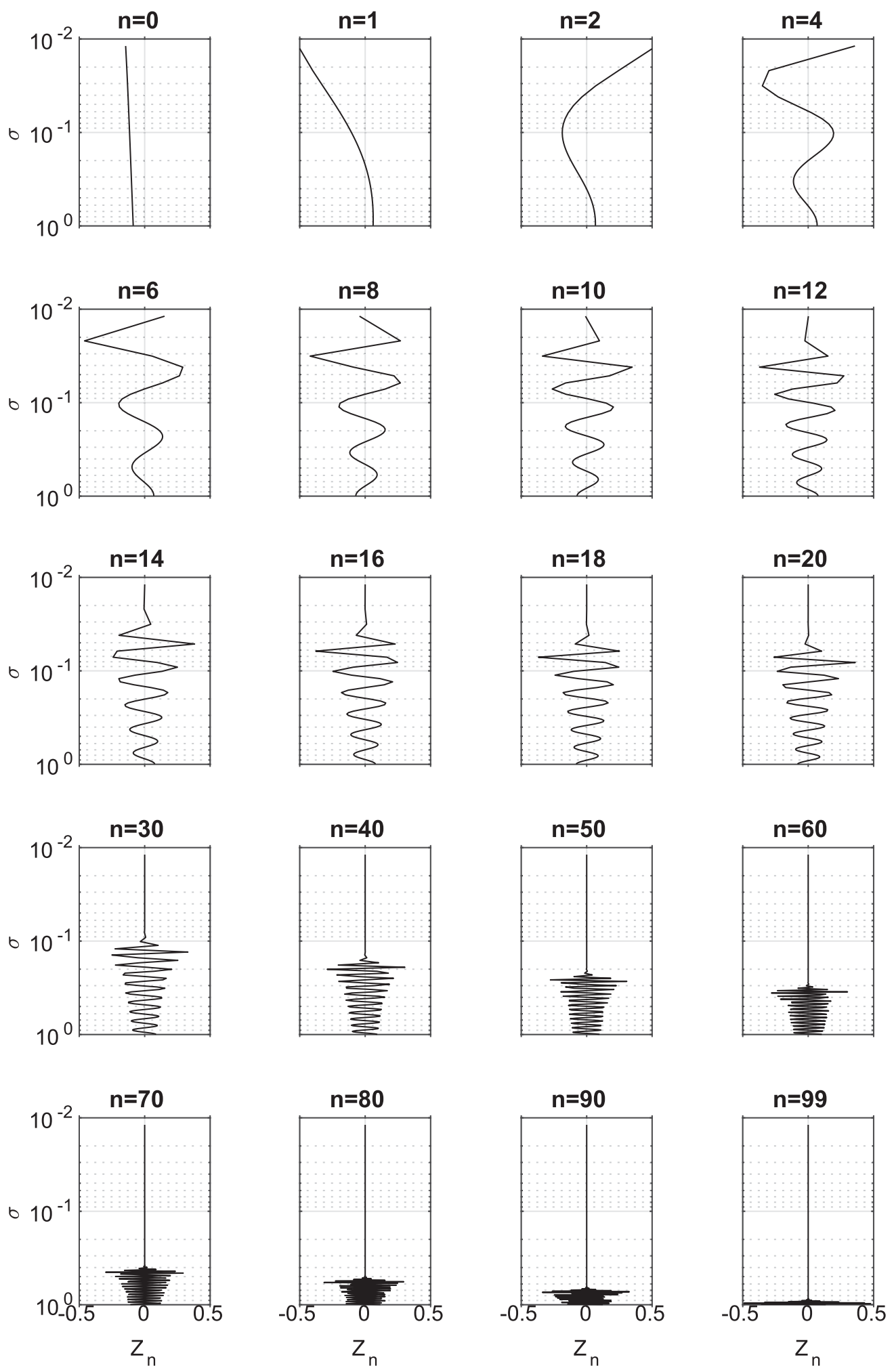

FIG. 4. Vertical structure of select vertical modes $Z_{n}(p)$. 

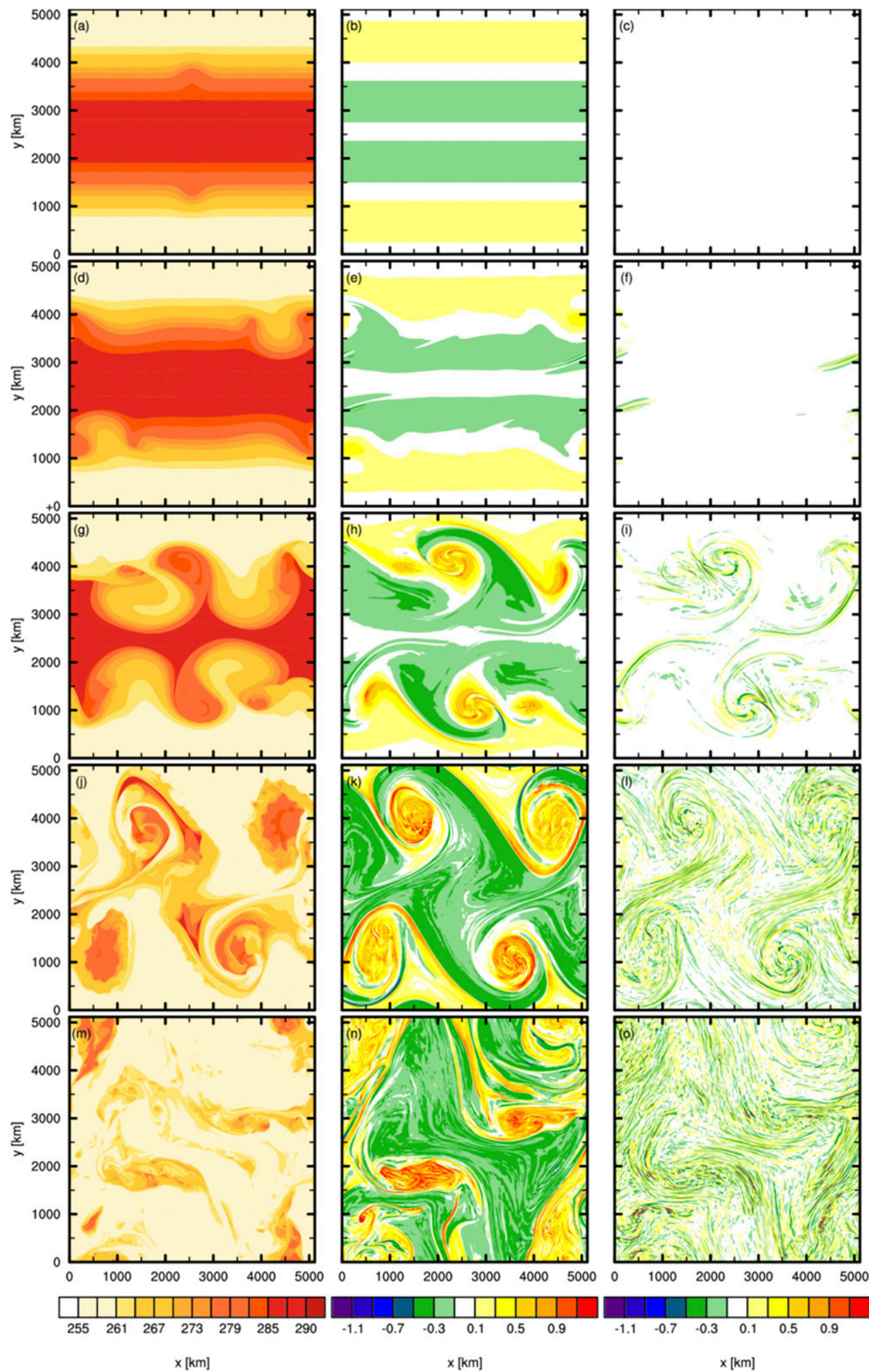

FIG. 5. Horizontal slices of (left) potential temperature $(K)$ in the lowest grid cell $(z \approx 42 \mathrm{~m})$, and (center) 500-hPa vertical vorticity, and (right) horizontal divergence at $t=$ (a)-(c) 0, (d)-(f) 4, (g)-(i) 8, (j)-(l) 12 , and $(\mathrm{m})-(\mathrm{o}) 16$ days. Vorticity and divergence are normalized by $f$. 

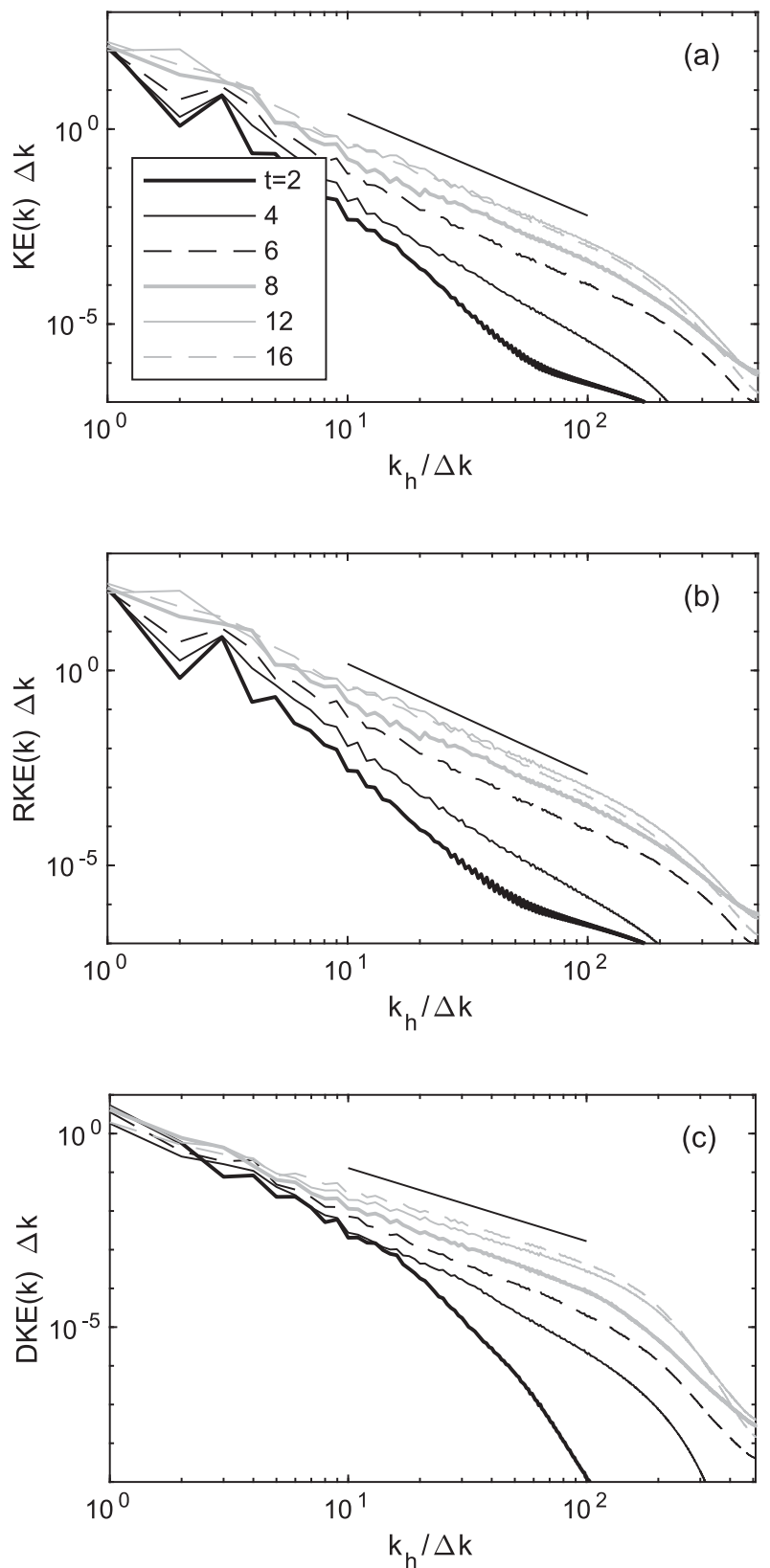

FIG. 6. Vertically averaged spectra of (a) kinetic energy, (b) rotational kinetic energy, and (c) divergent kinetic energy at $t=2,4,6,8,12$, and 16 days. The straight reference lines show the best-fit slope between $k=10$ and 100 at $t=16$ days: (a) -2.6 , (b) -2.8 , and (c) -1.9 . Here and in other spectra figures, a factor of $\Delta k$ is included so that the $y$ axis shows energy, not energy spectral density.

selection of times. By averaging spectra of energy per unit mass on model levels, the average spectra are proportional to energy per unit volume using the basic-state density (e.g., Waite and Snyder 2009). The kinetic energy spectrum (Fig. 6a) grows and fills out as the baroclinic instability develops. The spectrum saturates at around $t \approx 12$ days with a slope of -2.6 (unless otherwise stated, spectral slopes are measured between $k=10$ and 100 ). The spectrum falls off rapidly beyond $k \approx 200$ due to the numerical dissipation.

The RKE spectrum (Fig. 6b) resembles the total kinetic energy spectrum, and saturates with a slightly steeper slope of -2.8 . After 12 days, the RKE spectrum experiences a slight decrease in energy at large $k$. By contrast, the shallower DKE spectrum, which has a slope of -1.9 , continues to grow slowly in amplitude until the end of the simulation. The kinetic energy spectrum is the sum of the steeper RKE spectrum and shallower DKE spectrum; however, as in the dry baroclinic wave simulations of Waite and Snyder (2009), the amplitude of the DKE spectrum is not high enough to influence the total kinetic energy, which is dominated by the steeper RKE spectrum across all resolved wavenumbers. No mesoscale shallowing occurs in the vertically integrated KE spectrum. In what follows, we focus on $t=16$ days, since the DKE spectrum has the largest amplitude at this time.

Energy spectra computed on individual model levels are shown in Fig. 7. At $850 \mathrm{hPa}$ (Fig. 7a), the spectra resemble the vertical average, which is dominated by the lower troposphere: the RKE spectrum is steep, the DKE spectrum is shallow, and the total KE spectrum resembles the RKE over most wavenumbers. In fact, the vertically integrated $\mathrm{KE}$ spectrum (gray dashed curve in Fig. 7a) is nearly indistinguishable from the $850 \mathrm{hPa}$ KE spectrum. At $500 \mathrm{hPa}$ (Fig. 7b), the RKE and DKE spectra cross around $k \approx 60$, beyond which the DKE dominates and the kinetic energy spectrum shallows slightly. This transition is even more pronounced at $200 \mathrm{hPa}$, where the RKE and DKE spectra cross around $k \approx 25$ (Fig. 7c). Therefore, while the KE spectra in the lower stratosphere show clear mesoscale shallowing dominated by DKE (as found in other idealized baroclinic wave studies, for example, Waite and Snyder 2009), the vertically averaged spectra, which are dominated by the lower troposphere, do not.

\section{c. Modal spectra}

Figure 8 shows the geostrophic and ageostrophic energy spectra for a selection of vertical modes at $t=$ 16 days. For small mode numbers, the geostrophic energy dominates at all horizontal scales, and both the geostrophic and ageostrophic energy spectra are very steep, with slopes of around -4 (the spectral slopes are plotted against $n$ in Fig. 9). Indeed, for $n \leq 3$, the ageostrophic energy spectrum is actually steeper than the geostrophic. As $n$ increases, both spectra get shallower, and the ageostrophic spectra are consistently shallower than the geostrophic. For example, at $n=10$, 

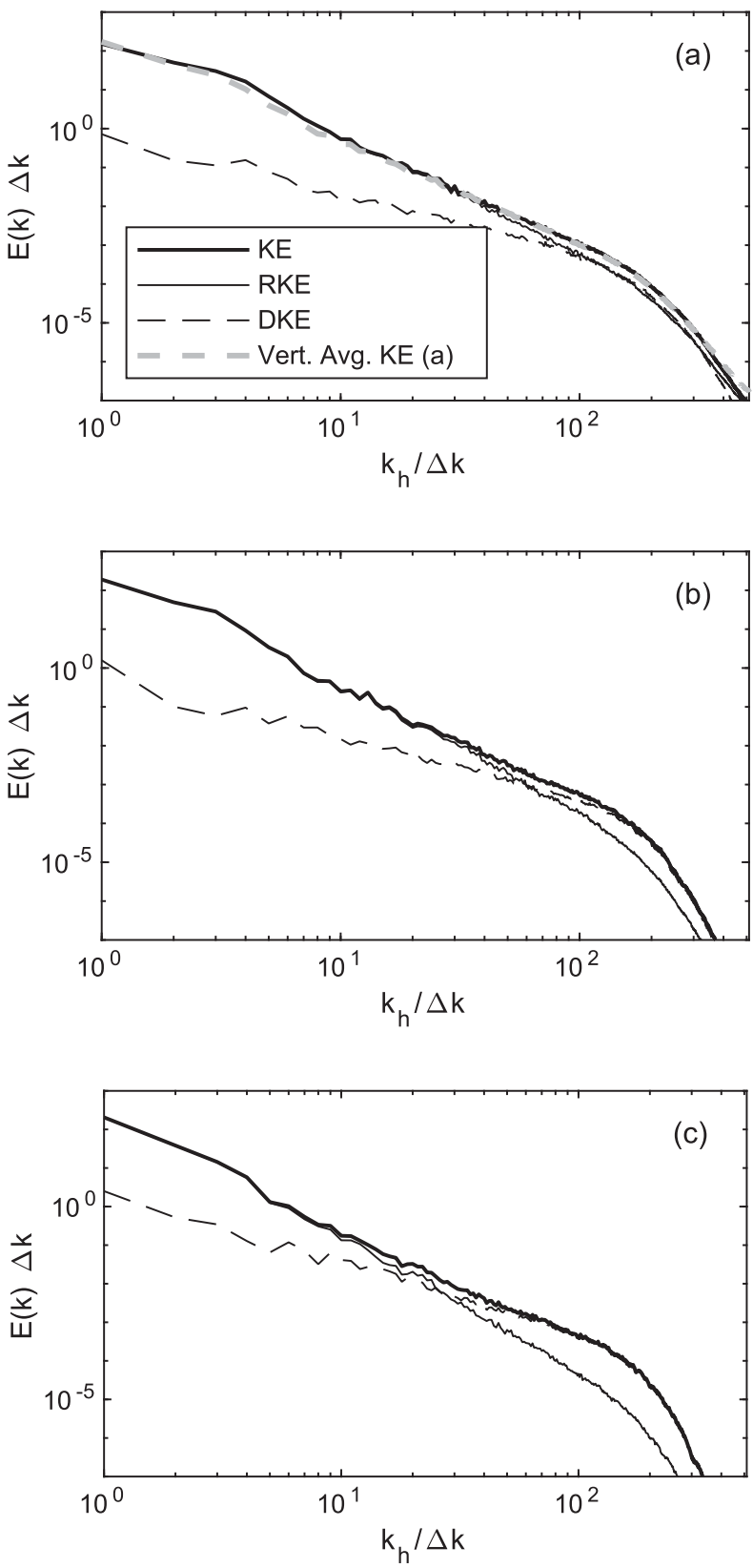

FIG. 7. Spectra of kinetic, rotational kinetic, and divergent kinetic energy at (a) 850 , (b) 500 , and (c) $200 \mathrm{hPa}$ at $t=16$ days. Spectra are computed on model levels, and pressures correspond to basic state. In (a), the vertically averaged KE spectrum is also shown by the gray dashed line for reference, and is almost entirely hidden by KE and RKE (solid thick and thin black curves).

the geostrophic and ageostrophic slopes are -3.3 and -2.6 ; for $n=20$, they are -3.0 and -2.0 ; and for $n=30$, they are -2.5 and -1.4 . As a result of their different slopes, the shallower ageostrophic spectrum intersects the steeper geostrophic spectrum for $n \geq 5$ and exceeds the GE at large $k$. The geostrophic energy spectral slope shallows to around -2 by $n=40$ and stays there for larger $n$, while the ageostrophic spectrum gets increasingly shallow for larger $n$ (see below).

The geostrophic energy spectrum is peaked at small wavenumbers for all $n$. The largest-scale geostrophic vortices project onto small $k$ at all $n$. By contrast, the peak in the ageostrophic spectrum moves to larger wavenumbers with increasing $n$. The wavenumber peak appears to occur near the deformation wavenumber, which is indicated by the vertical lines in Fig. 8. We can measure the peak by finding the wavenumber of maximum $k E_{A}(k, n)$ for each $n$; this wavenumber is plotted along with $k_{R}$ in Fig. 10 . The actual peak wavenumbers are indeed clustered around $k_{R}$ for $n \geqslant 10$. These spectra imply that inertia-gravity waves with increasing $n$, and therefore decreasing vertical scale, are characterized by a correspondingly small horizontal scale that is given by the deformation scale. For $n \geq 50$, the peak occurs near the numerical dissipation range; it cannot increase further, and no spectral power law is resolved at larger $k$.

Generally, the amplitudes of the spectra in Fig. 8, and the total amount of energy in each vertical mode, decrease with increasing $n$. The geostrophic and ageostrophic energy in each vertical mode are plotted in Fig. 11a. Most of the energy in the system is geostrophic energy in the lowest few modes, associated with the large-scale baroclinic wave, as expected. The geostrophic energy is peaked at $n=3$ and dominates over the ageostrophic energy for all $n$ [cf. Terasaki and Tanaka (2007), who similarly found a peak in the total energy at mode 4]. Interestingly, both the geostrophic and ageostrophic energy per mode decreases like $n^{-3}$ for $n \geqslant 10$, which is reminiscent of the -3 vertical wavenumber spectrum associated with QG turbulence. The mesoscale energy in each vertical mode is computed by restricting to $k \geq 10$ (Fig. 11b). The mesoscale geostrophic energy is peaked at $n=3$, like the total geostrophic energy, which is dominated by large horizontal scales. By contrast, the mesoscale ageostrophic energy is contained in intermediate vertical modes, and peaked at $n \approx 9$.

\section{d. Helmholtz decomposition}

The left column of Fig. 12 shows the geostrophic, rotational kinetic, and potential energy spectra for modes $n=1,10,20,40$, and 80. Equations (38) and (42) suggest that the geostrophic energy spectrum should be well approximated by the rotational kinetic energy spectrum for $k \gg k_{n}^{R}$ and by the potential energy spectrum for $k \ll k_{n}^{R}$. Figure 12 shows that these limiting spectra are approximately correct, although the transition does not happen right at $k_{R}^{n}$. For $n=1$, the deformation wavenumber is smaller than gravest wavenumber $k=1$ and 

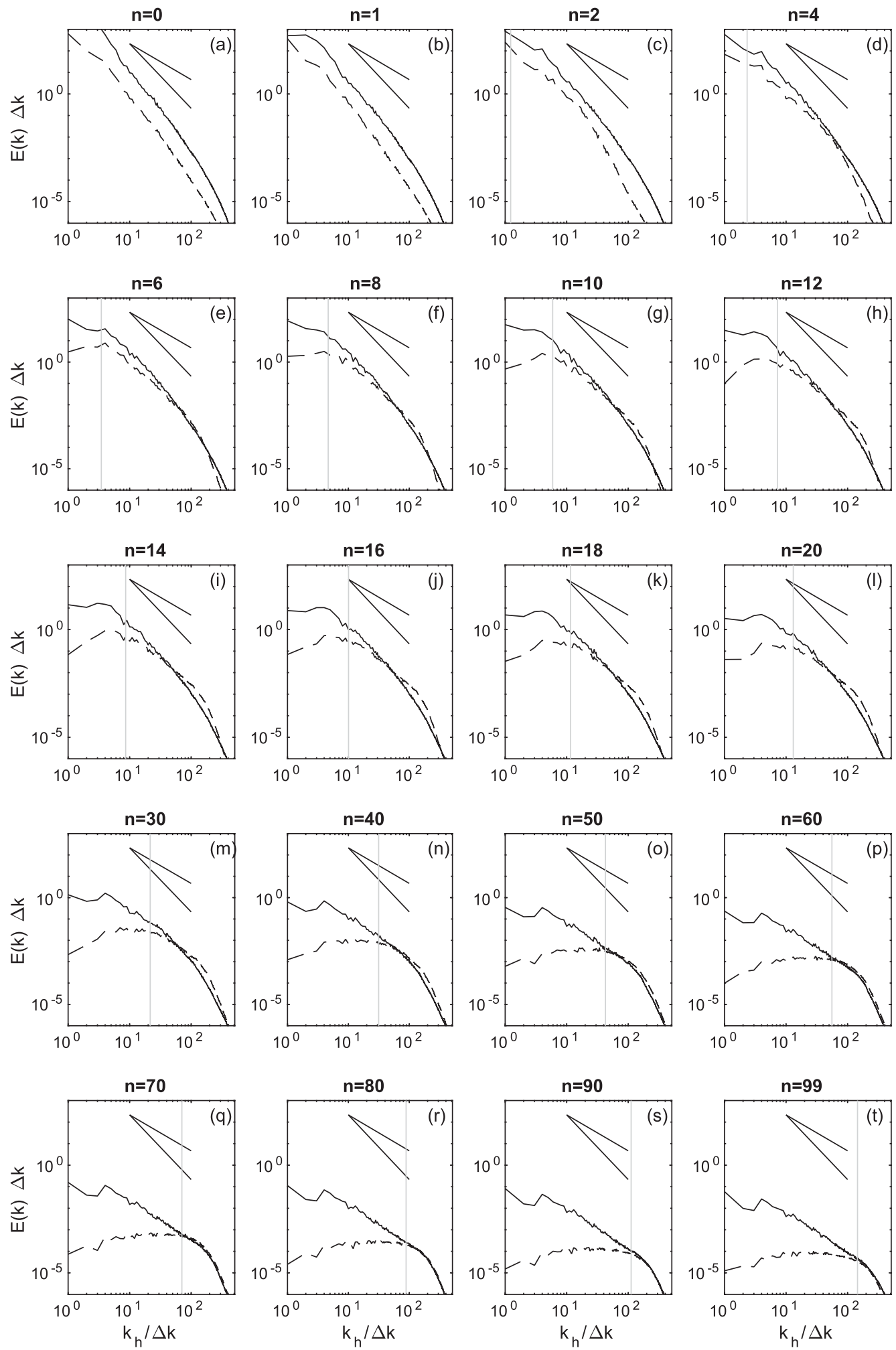

FIG. 8. Spectra of geostrophic (solid) and ageostrophic (dashed) energy at $t=16$ days for a selection of modes, as labeled (as in Fig. 3). The black reference curves have slopes of $-5 / 3$ and -3 , and the gray vertical line indicates the Rossby deformation wavenumber $k_{n}^{R}$. 


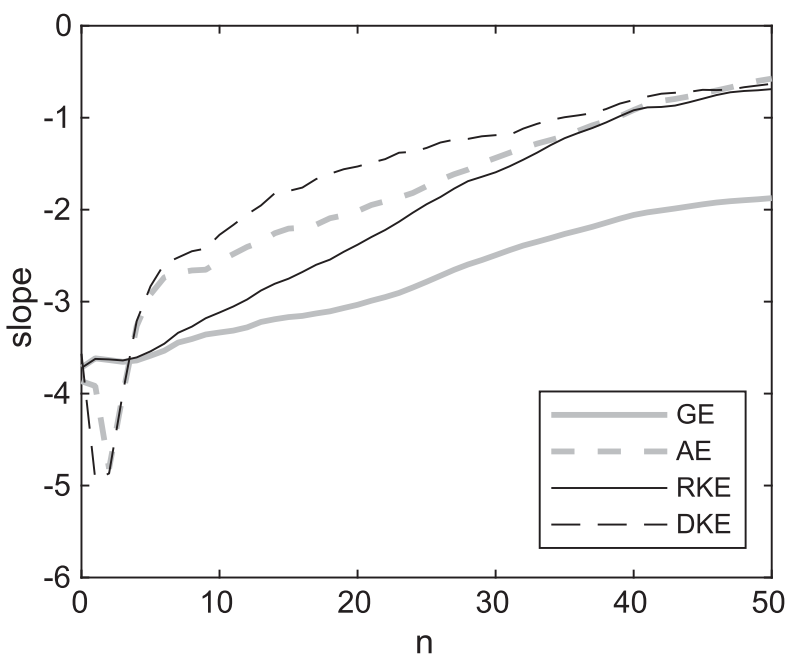

FIG. 9. Mesoscale slope of the geostrophic, ageostrophic, rotational kinetic, and divergent kinetic energy spectra at $t=16$ days. Slopes are measured for $10 \leq k \leq 100$.

the geostrophic energy spectrum is indeed indistinguishable from the RKE spectrum, as expected. For increasing $n$ and $k_{n}^{R}$, the GE and RKE spectra diverge at large scales, and we obtain $\operatorname{GE}(k, n) \approx \operatorname{PE}(k, n)$ for small $k$ and $\operatorname{GE}(k, n) \approx \operatorname{RKE}(k, n)$ for large $k$, also as expected.

However, the transition happens at $k$ larger than $k_{n}^{R}$. See, for example, $n=20$, for which $k_{R}^{n}=13$ : Fig. $12 \mathrm{e}$ shows $\operatorname{GE}(k, n) \approx \operatorname{PE}(k, n)$ for $k \lesssim 30$, and $\operatorname{GE}(k, n) \approx$ $\operatorname{RKE}(k, n)$ for $k \geq 80$. This finding is confirmed in Fig. 13a, which shows the spectral ratios $\operatorname{PE}(k, n) / \operatorname{GE}(k, n)$ and $\operatorname{RKE}(k, n) / \operatorname{GE}(k, n)$ with a linear $y$ axis: the transition between PE- and RKE-dominated geostrophic energy clearly happens at $k$ between 30 and 80 , which is a larger than $k_{n}^{R}$. The discrepancy with the prediction in Eqs. (38) and (42) is due to the fact that the potential energy is actually much larger than the rotational kinetic energy at $k=k_{n}^{R}$, in contrast to the assumption made in deriving Eq. (38). For $n=20$, the PE is 5 times larger than the RKE at this scale. Therefore, larger $k / k_{n}^{R}$ is required for the $\mathrm{PE}$ contribution to be negligible in Eq. (38), and similarly for Eq. (42). Nevertheless, the transition wavenumber clearly increases with $k_{R}^{n}$ as $n$ increases. For $n=80$ (Fig. 12i), the deformation scale is close to the numerical dissipation range, and $\operatorname{GE}(k, n) \approx$ $\mathrm{PE}(k, n)$ across all undamped scales. As a result of the dependence on $k_{n}^{R}$, the mesoscale slopes of the geostrophic and rotational kinetic energy spectra agree well for $n \lesssim 10$, but diverge at larger $n$, for which the RKE spectra are much shallower than the GE (Fig. 9).

The right column of Fig. 12 shows the ageostrophic, divergent kinetic, divergent kinetic plus potential, and kinetic energy spectra for the same modes as in the left

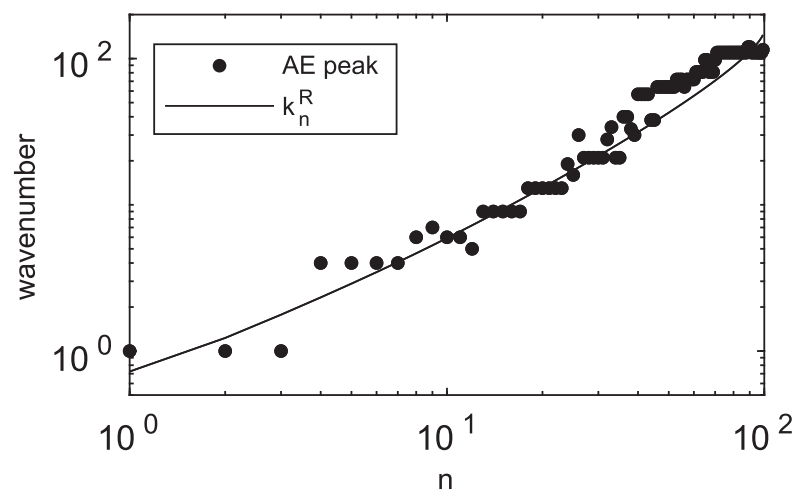

FIG. 10. The wavenumber of the peak in ageostrophic energy (black circles) and the Rossby deformation wavenumber (solid) plotted against $n$.

column. As was the case for the geostrophic energy, the ageostrophic energy spectra follow the approximations in Eqs. (43) and (46) at large and small wavenumbers: the ageostrophic energy is approximated by the potential plus divergent kinetic energy for $k \gg k_{n}^{R}$ and by the kinetic energy for $k \ll k_{n}^{R}$. For $n=1$, the ageostrophic energy is nearly identical to the potential plus divergent kinetic energy at all wavenumbers, which are all larger than $k_{1}^{R}$. Moving to larger $n$, our results show $\operatorname{AE}(k, n) \approx \operatorname{KE}(k, n)$ for small $k$ and $\operatorname{AE}(k, n) \approx$ $\operatorname{DKE}(k, n)+\operatorname{PE}(k, n)$ for large $k$, again as expected.

However, as was the case for the geostrophic energy spectra, the transition between these approximation regimes occurs at a wavenumber beyond $k_{n}^{R}$. For example, for $n=20$ (Fig. 12g), we have $\operatorname{AE}(k, n) \approx \operatorname{KE}(k, n)$ for $k \lesssim 30$ and $\operatorname{AE}(k, n) \approx \operatorname{DKE}(k, n)+\operatorname{PE}(k, n)$ for $k \gtrsim 80$; these are the same transition wavenumbers as for the geostrophic spectrum, as expected, and they are larger than $k_{20}^{R}=13$. The transition in the $n=20$ case is illustrated more clearly in Fig. 13b, which shows the ratios $\operatorname{KE}(k, n) / \operatorname{AE}(k, n)$ and $\operatorname{DKE}(k, n)+$ $\mathrm{PE}(k, n)] / \operatorname{AE}(k, n)$. Interestingly, while the large- $k$ approximation is excellent for $k>80$, the small- $k$ approximation is not great: the potential plus divergent kinetic energy underapproximates the ageostrophic energy at small $k$. This discrepancy is due to the fact that the potential energy is much larger than the kinetic energy at small $k$, and therefore very small $k / k_{R}^{n}$ are required for the PE term in Eq. (45) to be negligible. Similarly, the RKE is much larger than the DKE at $k_{n}^{R}$, so larger $k / k_{n}^{R}$ is required for the RKE term to be negligible in Eq. (45). Overall, the mesoscale slopes of the ageostrophic and divergent kinetic energy are much closer over all $n$ than those of the geostrophic and rotational kinetic energy, although the DKE spectra are slightly shallower for $10 \lesssim n \lesssim 40$. 

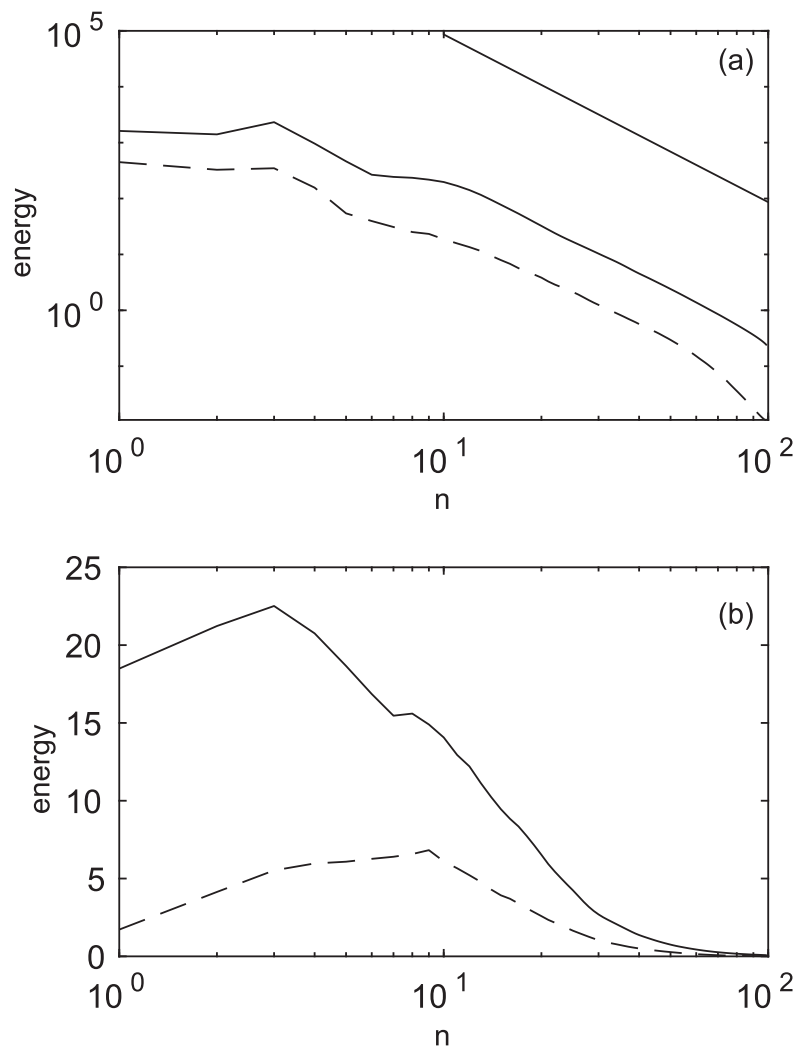

FIG. 11. Total geostrophic (solid) and ageostrophic (dashed) energy in each vertical mode, computed using (a) all horizontal scales and (b) mesoscales $(k \geq 10)$. The solid reference line has a slope of -3 .

\section{e. Vertically averaged geostrophic and ageostrophic spectra}

While the modal spectra allow for a detailed comparison of the geostrophic/ageostrophic and Helmholtzdecomposed spectra, most model spectral analysis considers vertically averaged (over layers if not the whole domain), not modal, spectra. Figure 14 shows modal spectra summed over all vertical modes, corresponding to a vertical average over the whole domain [cf. Eq. (34)]. The geostrophic and rotational kinetic energy spectra agree well over all $k$. There is slightly more GE than RKE at intermediate wavenumbers, but overall, the amplitudes and slopes are very similar: the mesoscale slopes of the geostrophic and rotational kinetic energy spectra are -3.0 and -2.8 , respectively. As discussed above, the RKE spectrum is expected to approximate the GE spectrum for $k$ larger than the Rossby deformation wavenumber; since the vertically averaged spectra are dominated by small $n$ (Fig. 11), which have relatively small $k_{n}^{R}$ (e.g., $0.7,1.2$, and 1.8 , for $n=1,2$, and 3 ), the agreement spans all $k$.
The ageostrophic and divergent kinetic energy spectra have similar slopes but different amplitudes over all $k$. The mesoscale slopes are -2.1 and -1.9 , respectively, for the $\mathrm{AE}$ and $\mathrm{DKE}$ spectra. The vertically averaged ageostrophic spectrum is shallower than the ageostrophic spectra of the dominant low vertical modes (Fig. 8); for example, the ageostrophic spectral slopes are $-3.9,-4.8$, and -3.3 for $n=1,2$, and 4 , which are all steeper than -2.1 . The shallowness of the vertically averaged ageostrophic spectrum seems to be due to the higher $n$ modes, the spectra of which are peaked at $k_{n}^{R}$ and are therefore increasingly flat for larger $n$. The ageostrophic energy spectrum has a larger amplitude and slightly steeper slope than the DKE spectrum: as a result, the gap between these spectra narrows with increasing $k$, from a factor of 8 at $k=1$, to 4 at $k=10$, and 2.5 at $k=11$. The DKE spectrum always underestimates the AE spectrum, by a factor of 2.5 at $k=100$, because of the missing contributions from the PE and (to a lesser degree) the RKE. Nevertheless, as with the GE and RKE spectra, the divergent kinetic energy spectral slope gives a good approximation of the ageostrophic spectral slope over all $k$ when vertically integrated spectra are considered.

\section{Conclusions}

Energy spectra in idealized baroclinic wave simulations were investigated using linear normal modes. The usual approach for analyzing energy spectra in atmospheric models is to consider kinetic energy spectra, often Helmholtz-decomposed into rotational and divergent KE, at different vertical levels. The normal mode approach is different: it decomposes the total domain-averaged mechanical energy into geostrophic and ageostrophic energy at every horizontal and vertical mode. In the idealized simulations performed here, the horizontal mode structure is Fourier and the vertical mode structure is found by solving the appropriate eigenvalue problem.

The vertically averaged KE spectra have a simple and familiar power-law structure: the RKE has a slope of around -2.8 and the DKE has a shallower slope of around -1.9 , which are close to the -3 and $-5 / 3$ reported elsewhere (e.g., Waite and Snyder 2009; Peng et al. 2015a). However, the amplitude of the DKE spectrum is too small to influence the kinetic energy spectrum, even at small scales. The KE spectral slope is -2.6 down to the (numerical) dissipation scale and no transition to a shallower mesoscale spectrum is apparent. As found in previous studies of idealized dry baroclinic waves (e.g., Waite and Snyder 2009), there is a transition to a shallower spectrum in the lower 

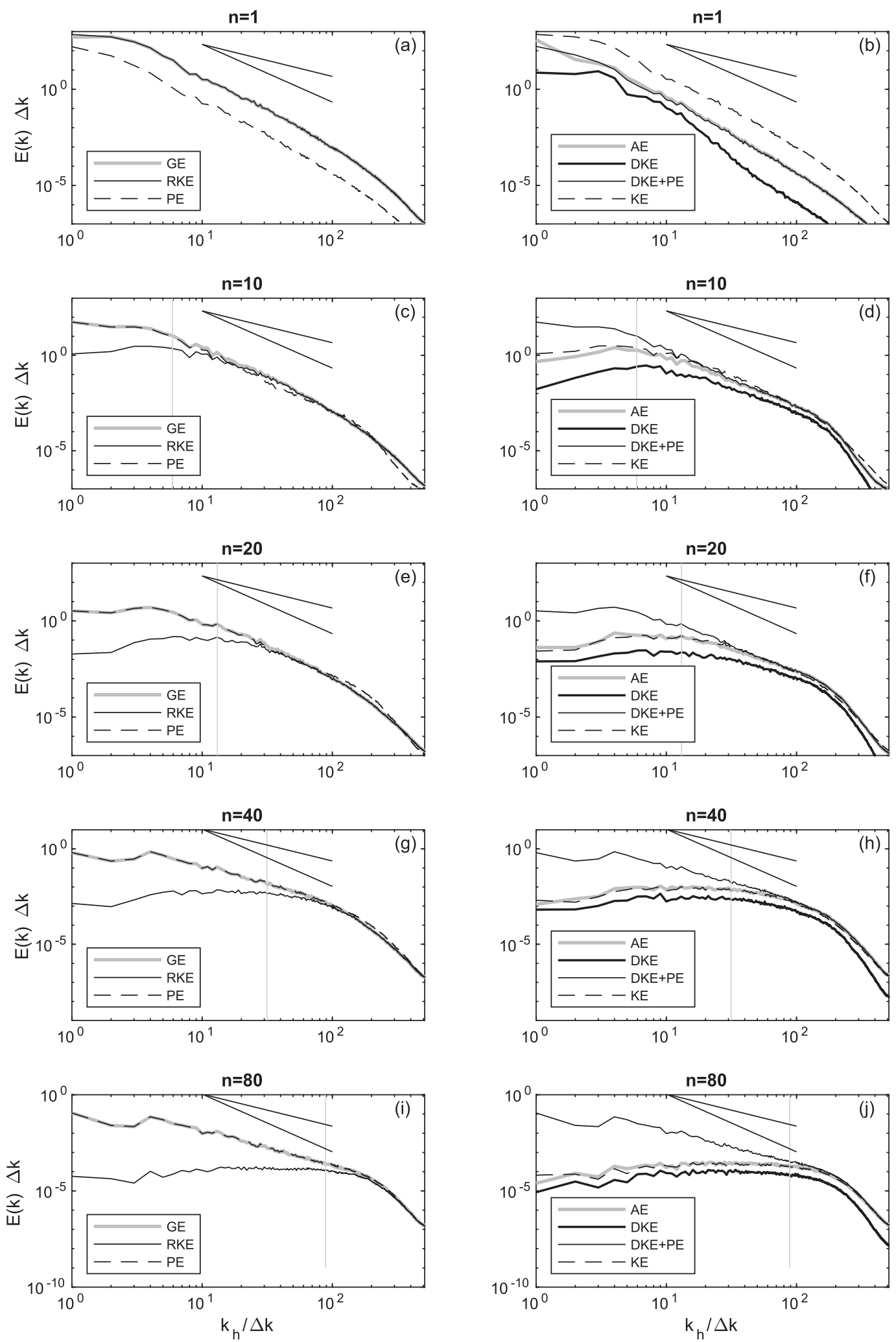

FIG. 12. (left) Spectra of geostrophic, rotational kinetic, and potential energy. (right) Spectra of ageostrophic, divergent kinetic, divergent kinetic plus potential, and kinetic energy. All spectra are at $t=16$ days for a selection of modes, as labeled. The black reference curves have slopes of $-5 / 3$ and -3 , and the gray vertical line indicates the Rossby deformation radius $k_{n}^{R}$. 

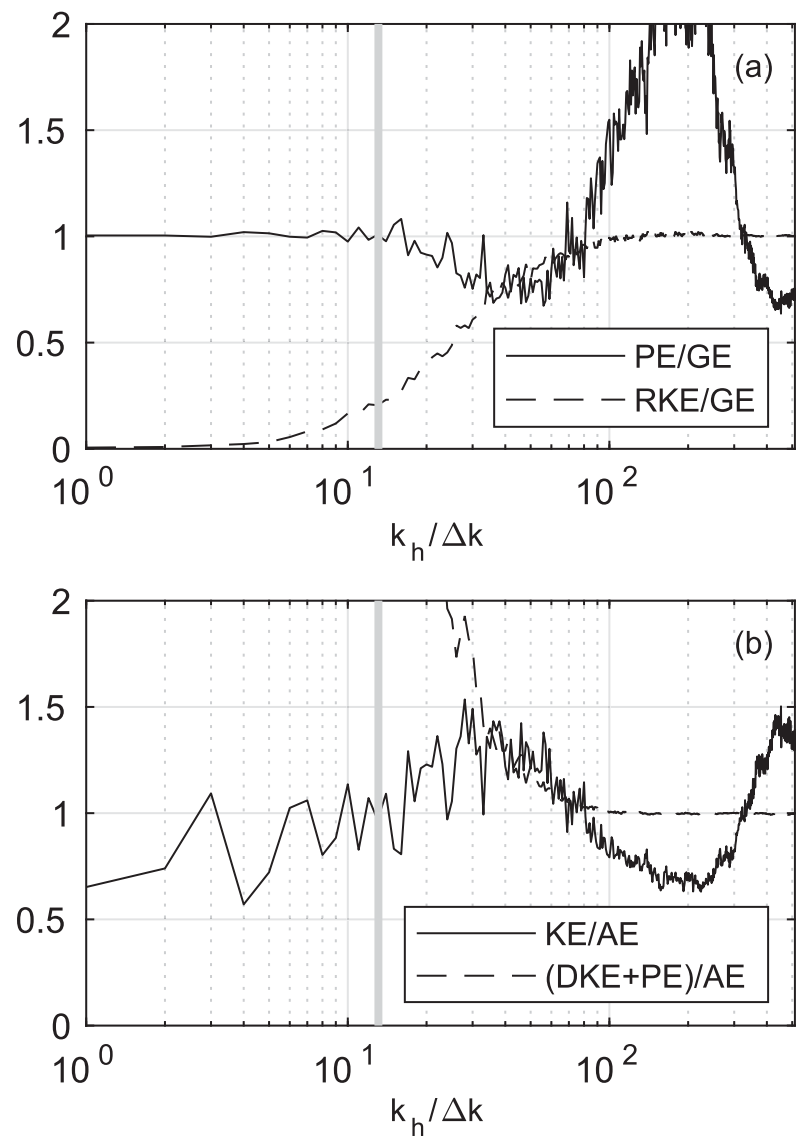

FIG. 13. (a) The ratio of the potential and rotational kinetic energy to the geostrophic energy and (b) the ratio of the kinetic and the potential plus divergent kinetic energy to the ageostrophic energy. In both panels, $n=20$ and the thick vertical gray line shows $k_{20}^{R}=13$.

stratosphere, but not in the troposphere; since the vertically averaged spectra are dominated by lower levels, this shallowing is not apparent in the vertical average.

The normal mode decomposition gives an interesting perspective into how the energy is partitioned into geostrophic and ageostrophic (IGW) motion at different horizontal and vertical scales. For small vertical mode numbers, corresponding to deep vertical structures, both the GE and AE spectra are very steep, and the GE energy dominates at all $k$. However, for increasing $n$, the $\mathrm{AE}$ spectrum shallows while the GE spectrum remains steep; for $n \geq 5$, the GE and AE spectra cross and the small horizontal scales are dominated by ageostrophic energy. Overall, both the GE and AE spectra get shallower with increasing $n$. The GE spectrum, which is always peaked at large scales, appears to be approaching a slope of around -2 . By contrast, the AE spectra are peaked around the Rossby deformation wavenumber $k_{n}^{R}$. As a result, for large $n$ with correspondingly large $k_{n}^{R}$, the AE spectra are nearly flat for a wide range of $k$.

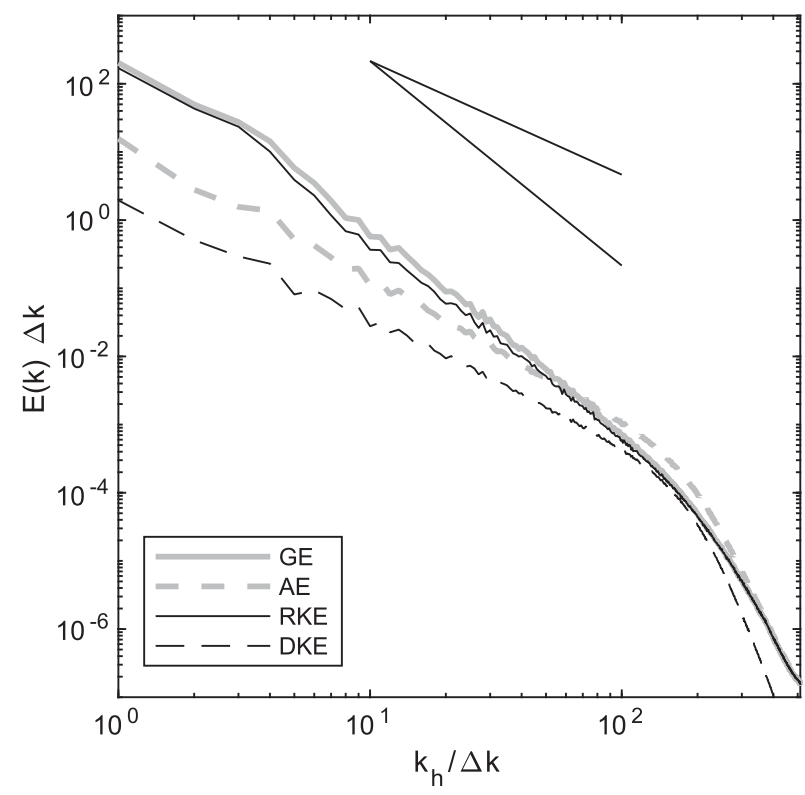

FIG. 14. Spectra of geostrophic, ageostrophic, rotational kinetic, and divergent kinetic energy summed over all $n$.

Most of the energy in the system, both GE and AE, is contained in the lowest vertical modes, which have the largest vertical scales. Indeed, the modal energy decreases with increasing $n$ like $n^{-3}$, similar to the Fourier spectrum expected for homogeneous QG turbulence. Since the energy in every vertical mode is dominated by the largest scales, it is perhaps not surprising that the vertical modal spectrum follows the QG scaling. However, when only the mesoscale $(k \geq 10)$ is considered, there is a significant difference between geostrophic and ageostrophic energy. The mesoscale geostrophic energy continues to be dominated by the smallest $n$, but the ageostrophic energy is peaked at $n=$ 9 , which has a midtropospheric vertical scale of a few kilometers.

A detailed comparison between the normal mode and Helmholtz decompositions was given. We showed that, for $k \gg k_{n}^{R}$, the geostrophic energy should be approximately given by the rotational kinetic energy, and the ageostrophic energy should be the sum of the divergent kinetic and potential energies. These limits provide a potential justification for the use of RKE and DKE as proxies for geostrophic and wave energy in the spectral analysis of data (e.g., Cho et al. 1999; Lindborg 2007) and simulations (e.g., Hamilton et al. 2008; Skamarock and Klemp 2008), at least in the midlatitude mesoscale [at larger scales and lower latitudes, velocity divergence in the geostrophic (Rossby) mode complicates this relationship]. In practice, these approximations were realized in our spectra, but the transition wavenumber was larger than $k_{n}^{R}$. As a result, for small $n$, the mesoscale GE 
and AE spectra were well approximated by the RKE and the sum of the DKE and PE; however, for larger $n$, this approximation was less valid. Nevertheless, since the total energy is dominated by smaller $n$, the Helmholtz decomposition actually performed very well when applied to the vertically averaged spectra: the RKE spectrum is very close to the GE spectrum over all $k$, while the slope of the DKE spectrum was a good approximation to that of the AE spectrum. However, since the AE spectrum also has a significant contribution from potential energy, which is missing in the Helmholtz decomposition, the DKE spectrum underapproximates the AE spectral amplitude by a factor of around 2.5. This factor could be important when using the spectral crossing wavenumber to diagnose the location of the mesoscale transition. The AE spectrum will cross the GE spectrum at a smaller wavenumber than that where the DKE spectrum crosses the RKE spectrum [e.g., compare Žagar et al. (2017), who considered GE-AE crossings, with Waite and Snyder (2009), who looked at RKE-DKE crossings; see also Deusebio et al. (2013), who show both in the idealized homogeneous case].

The similarity between the normal mode and Helmholtz decomposed spectral slopes is promising. The Helmholtz decomposition is simple and straightforward to employ for both aircraft and model data, and these results shown here suggest that the RKE and DKE spectral slopes are indeed good approximations to the more physically meaningful geostrophic and ageostrophic modal spectra. However, caution and more work are required before extrapolating these idealized results to more comprehensive models. In particular, simulations and reanalysis in larger or global domains, in which the Rossby deformation scales of the dominant vertical modes are smaller than the domain size, would likely show more of a difference between the normal mode and Helmholtz spectral slopes at large scales. Rossby wave velocity divergence associated with meridionally varying Coriolis parameter is also missing in the $f$-plane setup considered here. In addition, physical mechanisms that are neglected here are known to amplify the DKE spectra, and will therefore have a similar effect on the AE: these include moist physics (Waite and Snyder 2013; Peng et al. 2015a,b; Sun et al. 2017) and topography and surface fluxes (Menchaca and Durran 2019). Indeed, normal mode spectra from reanalysis data show that the GE and AE spectra do indeed cross at length scales of a few hundred kilometers, leading to a more pronounced mesoscale spectrum that what was found here (Terasaki et al. 2011; Žagar et al. 2017). The effects of these physical processes on the normal mode spectra, and in particular on the amplitude of the ageostrophic (IGW) energy, requires further investigation.
Acknowledgments. Model data used in this study are available upon request to the corresponding author. This work benefited from comments by Francis Poulin, Marek Stastna, and two anonymous referees. Computational resources were provided by the Shared Hierarchical Academic Research Computing Network (SHARCNET) and Compute/Calcul Canada. Funding from the Natural Sciences and Engineering Research Council of Canada (Grant RGPIN-386456-2015) is gratefully acknowledged.

\section{REFERENCES}

Augier, P., and E. Lindborg, 2013: A new formulation of the spectral energy budget of the atmosphere, with application to two high-resolution general circulation models. J. Atmos. Sci., 70, 2293-2308, https://doi.org/10.1175/JAS-D-12-0281.1.

Bartello, P., 1995: Geostrophic adjustment and inverse cascades in rotating stratified turbulence. J. Atmos. Sci., 52, 44104428, https://doi.org/10.1175/1520-0469(1995)052<4410: $\mathrm{GAAICI}>2.0 . \mathrm{CO} ; 2$.

Callies, J., O. Bühler, and R. Ferrari, 2016: The dynamics of mesoscale winds in the upper troposphere and lower stratosphere. J. Atmos. Sci., 73, 4853-4872, https://doi.org/10.1175/ JAS-D-16-0108.1.

Charney, J. G., 1971: Geostrophic turbulence. J. Atmos. Sci., 28, 1087-1095, https://doi.org/10.1175/1520-0469(1971)028<1087: $\mathrm{GT}>2.0 . \mathrm{CO} ; 2$.

Cho, J. Y. N., R. E. Newell, and J. D. Barrick, 1999: Horizontal wavenumber spectra of winds, temperature, and trace gases during the Pacific Exploratory Missions: 2. Gravity waves, quasi-two-dimensional turbulence, and vortical modes. J. Geophys. Res., 104, 16 297-16308, https://doi.org/10.1029/ 1999JD900068.

Cohn, S. E., and D. P. Dee, 1989: An analysis of the vertical structure equation for arbitrary thermal profiles. Quart. J. Roy. Meteor. Soc., 115, 143-171, https://doi.org/10.1002/ qj.49711548508.

Daley, R., 1991: Atmospheric Data Analysis. Cambridge University Press, $457 \mathrm{pp}$.

Deusebio, E., A. Vallgren, and E. Lindborg, 2013: The route to dissipation in strongly stratified and rotating flows. J. Fluid Mech., 720, 66-103, https://doi.org/10.1017/jfm.2012.611.

Dewan, E. M., 1979: Stratospheric wave spectra resembling turbulence. Science, 204, 832-835, https://doi.org/10.1126/ science.204.4395.832.

Gage, K. S., 1979: Evidence for a $k^{-5 / 3}$ law inertial range in mesoscale two-dimensional turbulence. J. Atmos. Sci., 36, 1950-1954, https://doi.org/10.1175/1520-0469(1979)036<1950: EFALIR $>2.0 . \mathrm{CO} ; 2$.

Hamilton, K., Y. O. Takahashi, and W. Ohfuchi, 2008: Mesoscale spectrum of atmospheric motions investigated in a very fine resolution global general circulation model. J. Geophys. Res., 113, D18110, https://doi.org/10.1029/2008JD009785.

Herbert, C., R. Marino, D. Rosenberg, and A. Pouquet, 2016: Waves and vortices in the inverse cascade regime of stratified turbulence with or without rotation. J. Fluid Mech., 806, 165204, https://doi.org/10.1017/jfm.2016.581.

Kafiabad, H. A., and P. Bartello, 2016: Balance dynamics in rotating stratified turbulence. J. Fluid Mech., 795, 914-949, https://doi.org/10.1017/jfm.2016.164. 
Kasahara, A., 1976: Normal modes of ultralong waves in the atmosphere. Mon. Wea. Rev., 104, 669-690, https:// doi.org/10.1175/1520-0493(1976)104<0669:NMOUWI> 2.0.CO;2.

-_, and K. Puri, 1981: Spectral representation of threedimensional global data by expansion in normal mode functions. Mon. Wea. Rev., 109, 37-51, https://doi.org/10.1175/ 1520-0493(1981)109<0037:SROTDG > 2.0.CO;2.

——, and J.-H. Qian, 2000: Normal modes of a global nonhydrostatic atmospheric model. Mon. Wea. Rev., 128, 3357-3375, https://doi.org/10.1175/1520-0493(2000)128<3357: NMOAGN $>2.0 . \mathrm{CO} ; 2$.

Kitamura, Y., and Y. Matsuda, 2010: Energy cascade processes in rotating stratified turbulence with application to the atmospheric mesoscale. J. Geophys. Res., 115, D11104, https:// doi.org/10.1029/2009JD012368.

Koshyk, J. N., and K. Hamilton, 2001: The horizontal kinetic energy spectrum and spectral budget simulated by a highresolution troposphere-stratosphere-mesosphere GCM. J. Atmos. Sci., 58, 329-348, https://doi.org/10.1175/1520-0469(2001) $058<0329$ :THKESA $>2.0 . \mathrm{CO} ; 2$.

Li, Q., and E. Lindborg, 2018: Weakly or strongly nonlinear mesoscale dynamics close to the tropopause. J. Atmos. Sci., 75, 1215-1229, https://doi.org/10.1175/JAS-D-17-0063.1.

Lilly, D. K., 1983: Stratified turbulence and the mesoscale variability of the atmosphere. J. Atmos. Sci., 40, 749-761, https://doi.org/10.1175/1520-0469(1983)040<0749:STATMV> 2.0. $\mathrm{CO} ; 2$

Lindborg, E., 2006: The energy cascade in a strongly stratified fluid. J. Fluid Mech., 550, 207-242, https://doi.org/10.1017/ S0022112005008128.

_ 2007: Horizontal wavenumber spectra of vertical vorticity and horizontal divergence in the upper troposphere and lower stratosphere. J. Atmos. Sci., 64, 1017-1025, https://doi.org/ 10.1175/JAS3864.1.

— tions in the upper troposphere and lower stratosphere: 2. Theoretical considerations. J. Geophys. Res., 106, 10233 10241, https://doi.org/10.1029/2000JD900815.

Malardel, S., and N. P. Wedi, 2016: How does subgrid-scale parametrization influence nonlinear spectral energy fluxes in global NWP models? J. Geophys. Res. Atmos., 121, 5395-5410, https://doi.org/10.1002/2015JD023970.

Menchaca, M. Q., and D. R. Durran, 2019: The influence of gravity waves on the slope of the kinetic-energy spectrum in simulations of idealized midlatitude cyclones. J. Atmos. Sci., 76, 2103-2122, https://doi.org/10.1175/JAS-D-18-0329.1.

Nastrom, G. D., and K. S. Gage, 1985: A climatology of atmospheric wavenumber spectra observed by commercial aircraft. J. Atmos. Sci., 42, 950-960, https://doi.org/10.1175/15200469(1985)042<0950:ACOAWS > 2.0.CO;2.

Peng, J., L. Zhang, and J. Guan, 2015a: Applications of a moist nonhydrostatic formulation of the spectral energy budget to baroclinic waves. Part I: The lower-stratospheric energy spectra. J. Atmos. Sci., 72, 2090-2108, https://doi.org/10.1175/ JAS-D-14-0306.1.

, — - and - 2015b: Applications of a moist nonhydrostatic formulation of the spectral energy budget to baroclinic waves. Part II: The upper-tropospheric energy spectra. J. Atmos. Sci., 72, 3923-3939, https://doi.org/10.1175/JAS-D14-0359.1.

Riley, J. J., and E. Lindborg, 2008: Stratified turbulence: A possible interpretation of some geophysical turbulence measurements. J. Atmos. Sci., 65, 2416-2424, https://doi.org/ 10.1175/2007JAS2455.1.

Schaefer-Rolffs, U., and E. Becker, 2013: Horizontal momentum diffusion in GCMs using the dynamic Smagorinsky model. Mon. Wea. Rev., 141, 887-899, https://doi.org/10.1175/MWRD-12-00101.1.

Shutts, G., 2005: A kinetic energy backscatter algorithm for use in ensemble prediction systems. Quart. J. Roy. Meteor. Soc., 131 3079-3102, https://doi.org/10.1256/qj.04.106.

Skamarock, W. C., 2004: Evaluating mesoscale NWP models using kinetic energy spectra. Mon. Wea. Rev., 132, 3019-3032, https://doi.org/10.1175/MWR2830.1.

—, and J. B. Klemp, 2008: A time-split nonhydrostatic atmospheric model for weather research and forecasting applications. J. Comput. Phys., 227, 3465-3485, https://doi.org/10.1016/ j.jcp.2007.01.037.

— , and Coauthors, 2008: A description of the Advanced Research WRF version 3. NCAR Tech. Note NCAR/TN 475+STR, 113 pp., https://doi.org/10.5065/D68S4MVH.

—, S.-H. Park, J. B. Klemp, and C. Snyder, 2014: Atmospheric kinetic energy spectra from global high-resolution nonhydrostatic simulations. J. Atmos. Sci., 71, 4369-4381, https:// doi.org/10.1175/JAS-D-14-0114.1.

— C. Snyder, J. B. Klemp, and S.-H. Park, 2019: Vertical resolution requirements in atmospheric simulation. Mon Wea. Rev., 147, 2641-2656, https://doi.org/10.1175/MWR-D19-0043.1.

Staniforth, A., M. Béland, and J. Côté, 1985: An analysis of the vertical structure equation in sigma coordinates. Atmos.Ocean, 23, 323-358, https://doi.org/10.1080/07055900. 1985.9649232.

Sun, Y. Q., R. Rotunno, and F. Zhang, 2017: Contributions of moist convection and internal gravity waves to building the atmospheric $-5 / 3$ kinetic energy spectra. J. Atmos. Sci., 74, 185-201, https://doi.org/10.1175/JAS-D-16-0097.1.

Tanaka, H., 1985: Global energetics analysis by expansion into three-dimensional normal mode functions during the FGGE winter. J. Meteor. Soc. Japan, 63, 180-200, https://doi.org/ 10.2151/jmsj1965.63.2_180.

Terasaki, K., and H. L. Tanaka, 2007: An analysis of the 3-D atmospheric energy spectra and interactions using analytical vertical structure functions and two reanalyses. J. Meteor. Soc. Japan, 85, 785-796, https://doi.org/10.2151/jmsj.85.785.

$\ldots, \ldots$, and N. Žagar, 2011: Energy spectra of Rossby and gravity waves. SOLA, 7, 45-48, https://doi.org/10.2151/ SOLA.2011-012.

Tulloch, R., and K. S. Smith, 2006: A theory for the atmospheric energy spectrum: Depth-limited temperature anomalies at the tropopause. Proc. Natl. Acad. Sci. USA, 103, 14 690-14 694, https://doi.org/10.1073/pnas.0605494103.

Ullrich, P. A., K. A. Reed, and C. Jablonowski, 2015: Analytical initial conditions and an analysis of baroclinic instability waves in $f$ - and $\beta$-plane 3D channel models. Quart. J. Roy. Meteor. Soc., 141, 2972-2988, https://doi.org/10.1002/qj.2583.

VanZandt, T. E., 1982: A universal spectrum of buoyancy waves in the atmosphere. Geophys. Res. Lett., 9, 575-578, https:// doi.org/10.1029/GL009i005p00575.

Waite, M. L., 2016: Dependence of model energy spectra on vertical resolution. Mon. Wea. Rev., 144, 1407-1421, https:// doi.org/10.1175/MWR-D-15-0316.1.

— and C. Snyder, 2009: The mesoscale kinetic energy spectrum of a baroclinic life cycle. J. Atmos. Sci., 66, 883-901, https:// doi.org/10.1175/2008JAS2829.1. 
_ and - 2013: Mesoscale energy spectra of moist baroclinic waves. J. Atmos. Sci., 70, 1242-1256, https://doi.org/10.1175/ JAS-D-11-0347.1.

Warn, T., 1986: Statistical mechanical equilibria of the shallow water equations. Tellus, 38A, 1-11, https://doi.org/10.1111/ j.1600-0870.1986.tb00448.x.

Wicker, L. J., and W. C. Skamarock, 2002: Time-splitting methods for elastic models using forward time schemes. Mon. Wea. Rev., 130, 2088-2097, https://doi.org/10.1175/1520-0493(2002) $130<2088$ :TSMFEM>2.0.CO;2.
Wiin-Nielsen, A., 1971: On the motion of various vertical modes of transient, very long waves: Part I. Beta plane approximation. Tellus, 23, 87-98, https://doi.org/10.3402/TELLUSA.V23I1.10309.

Žagar, N., K. Terasaki, and H. L. Tanaka, 2012: Impact of the vertical resolution of analysis data on the estimates of largescale inertio-gravity energy. Mon. Wea. Rev., 140, 2297-2307, https://doi.org/10.1175/MWR-D-11-00103.1.

, D. Jelić, and M. Blaauw, 2017: Energy spectra and inertiagravity waves in global analyses. J. Atmos. Sci., 74, 2447-2466, https://doi.org/10.1175/JAS-D-16-0341.1. 